\title{
Combining next-generation sequencing and single-molecule sequencing to explore brown plant hopper responses to contrasting genotypes of japonica rice
}

Jing Zhang, Wei Guan, Chaomei Huang, Yinxia Hu, Yu Chen, Jianping Guo, Cong Zhou, Rongzhi Chen, Bo Du, Lili Zhu, Danax Huanhan and Guangcun He*

\begin{abstract}
Background: The brown plant hopper (BPH), Nilaparvata lugens, is one of the major pest of rice (Oryza sativa). Plant defenses against insect herbivores have been extensively studied, but our understanding of insect responses to host plants' resistance mechanisms is still limited. The purpose of this study is to characterize transcripts of BPH and reveal the responses of BPH insects to resistant rice at transcription level by using the advanced molecular techniques, the next-generation sequencing (NGS) and the single-molecule, real-time (SMRT) sequencing.

Results: The current study obtained 24,891 collapsed isoforms of full-length transcripts, and 20,662 were mapped to known annotated genes, including 17,175 novel transcripts. The current study also identified 915 fusion genes, 1794 novel genes, 2435 long non-coding RNAs (IncRNAs), and 20,356 alternative splicing events. Moreover, analysis of differentially expressed genes (DEGs) revealed that genes involved in metabolic and cell proliferation processes were significantly enriched in up-regulated and down-regulated sets, respectively, in BPH fed on resistant rice relative to $\mathrm{BPH}$ fed on susceptible wild type rice. Furthermore, the FoxO signaling pathway was involved and genes related to BPH starvation response (N/bmm), apoptosis and autophagy (caspase 8, ATG13, BNIP3 and IAP), active oxygen elimination (catalase, MSR, ferritin) and detoxification (GST, CarE) were up-regulated in BPH responses to resistant rice.

Conclusions: The current study provides the first demonstrations of the full diversity and complexity of the BPH transcriptome, and indicates that BPH responses to rice resistance, might be related to starvation stress responses, nutrient transformation, oxidative decomposition, and detoxification. The current result findings will facilitate further exploration of molecular mechanisms of interaction between BPH insects and host rice.
\end{abstract}

Keywords: Brown plant hopper, Host resistance, RNA-seq, Single-molecule, real-time sequencing, Transcriptome, FoxO signaling pathway

\footnotetext{
* Correspondence: gche@whu.edu.cn

State Key Laboratory of Hybrid Rice, College of Life Sciences, Wuhan

University, Wuhan, China
}

(c) The Author(s). 2019 Open Access This article is distributed under the terms of the Creative Commons Attribution 4.0 International License (http://creativecommons.org/licenses/by/4.0/), which permits unrestricted use, distribution, and reproduction in any medium, provided you give appropriate credit to the original author(s) and the source, provide a link to the Creative Commons license, and indicate if changes were made. The Creative Commons Public Domain Dedication waiver (http://creativecommons.org/publicdomain/zero/1.0/) applies to the data made available in this article, unless otherwise stated. 


\section{Background}

During more than 350 million years of co-evolution, herbivorous insects have developed various mechanisms to feed on plants, and counter diverse direct and indirect defenses against their feeding that plants have evolved [1-3]. Piercing and sucking insects comprise a large group of herbivores using plant phloem as their target and the transported fluid as their primary nutrient source. While feeding, these insects also reportedly secrete saliva into the plant tissue, which is thought to contain enzymes or effectors that interfere with plant defense responses [4]. Resistance and defense response of plants to piercing and sucking insects, which have been extensively studied, include systems that recognize effectors and trigger a stronger resistance reaction against herbivores. Sealing sieve tube, strengthening cell wall, and producing secondary metabolites and defenserelated proteins are major resistance mechanisms against phloem-sucking insects. Changes in membrane potentials, $\mathrm{Ca}^{2+}$-signaling, levels and distributions of reactive oxygen species, kinase activities and phytohormones are known to play essential roles in early plant immune response to insect [5-8]. However, our understanding of insect responses to resistant host plants, particularly at genomic and transcriptomic levels, is still limited.

The brown plant hopper (BPH), Nilaparvata lugens, is a piercing and sucking insect pest of rice (Oryza sativa) which belongs to family Delphacidae, order Hemiptera, class Insecta of phylum Arthropoda. BPH feeding causes damages directly to rice plants mainly during crop reproductive stage. When rice crop is seriously harmed by $\mathrm{BPH}$, the phenomenon of "hopper burn" occurs, resulting in significant reduction in rice yield, or even complete losses. $\mathrm{BPH}$ also indirectly injures rice plants by transmitting viruses $[9,10]$. As well as being a major crop, rice is a model plant for genome research [11]. Hence, multiple omics approaches, such as functional genomic, transcriptomic, proteomic, and metabonomic analyses have been used in attempts to elucidate rice defenses to $\mathrm{BPH}$ [12-14]. Several $\mathrm{BPH}$-resistance genes in rice have been isolated via the map-based cloning [15-18]. For example, Bph6 (which confers broad resistance to $\mathrm{BPH}$ and white-backed plant hopper) has been shown to encode a previously uncharacterized protein that interacts with the exocyst subunit OsEXO70E1, thereby increasing exocytosis, and induces coordinated activation of cytokinin, salicylic acid, and jasmonic acid signaling pathways in response to BPH feeding [16].

With the rapid development of sequencing technology, research on insect omics is increasing $[19,20]$. Highthroughput, highly scalable, next-generation sequencing now allows entire genomes or transcriptomes to be sequenced simultaneously, at feasible cost [21]. Moreover, single-molecule, real-time (SMRT) sequencing can provide full-length transcript information and accurate isoform annotation [22, 23], thereby enabling comprehensive analysis of complex transcriptomes [24-27]. Thus, as reported here, the current study have combined next-generation and SMRT sequencing to characterize transcripts of $\mathrm{BPH}$ (including tissues of antenna, stylet and salivary gland, which play crucial roles in interactions with host plants $[28,29])$ and reveal the responses of $\mathrm{BPH}$ insects to resistant rice by comparing insects fed on resistant Bph6-transgenic and susceptible wild type rice plants.

\section{Results \\ Sequencing and characterizing full-length isoforms of BPH}

SMRTbell libraries from mixed tissues of 80 female adults of BPH biotype 1 were sequenced using a PacBio Sequel system with a V2 reagent kit and three SMRT cells, yielding $15.69 \mathrm{~Gb}$ clean data. The insert length distribution in each cell was very similar (Fig. 1a, Table 1). The current study obtained 436,057 reads of inserts (ROI), with 11.31 passes on average. In total, 376,347 (86.31\%) reads were classified as full-length (FL) based on the presence of barcoded primers and poly A tails (Table 1). After Iterative Clustering for Error Correction (ICE) [30] followed by Long Read DBG Error Correction (Lordec) [31] using the current study RNA sequencing (RNA-Seq) data (see below) (Additional file 1: Figure S1), 176,807 sequences totaling 574,066,263 bp were obtained. These sequences were mapped to the $\mathrm{BPH}$ reference genome NilLug1.0 (https://www.ncbi.nlm.nih.gov/genome/?term=txid108931 [Organism:noexp]) using the Genomic Mapping and Alignment Program (GMAP) [32]. 172,329 (98.94\%) sequences were aligned to the $\mathrm{BPH}$ reference genome, and 2631 were identified as reads of 915 fusion genes. The current study obtained 24,891 collapsed isoforms of fulllength transcripts after redundancy removal, of which 20 , 662 were mapped to 7395 annotated genes in the reference genome. Of the 20,662 mapped transcripts, 3487 (16.88\%) were identical to the original transcripts, and most $(17,175,83.12 \%)$ were novel transcripts of known genes in $\mathrm{BPH}$ genome.

According to the current study PacBio data, 3749 of the 7395 annotated genes (50.70\%) encoded more than one isoform, compared to 2545 (12.85\% in 19,806 protein-coding genes) in the reference genome NilLug1.0. The average number of isoforms per gene (2.79) in the PacBio dataset was more than twice the number (1.23) in the reference genome (Fig. 1b). In addition, 237 of the 7395 annotated genes (3.20\%) had more than 10 isoforms, and three had extremely high numbers of isoforms: PB.6723 (LOC111054078, 353 isoforms), PB.450 (LOC111049488, 326 isoforms), and PB.5698 (LOC111051863, 298 isoforms). Furthermore, the number of isoforms per gene was positively correlated with the number of exons per 

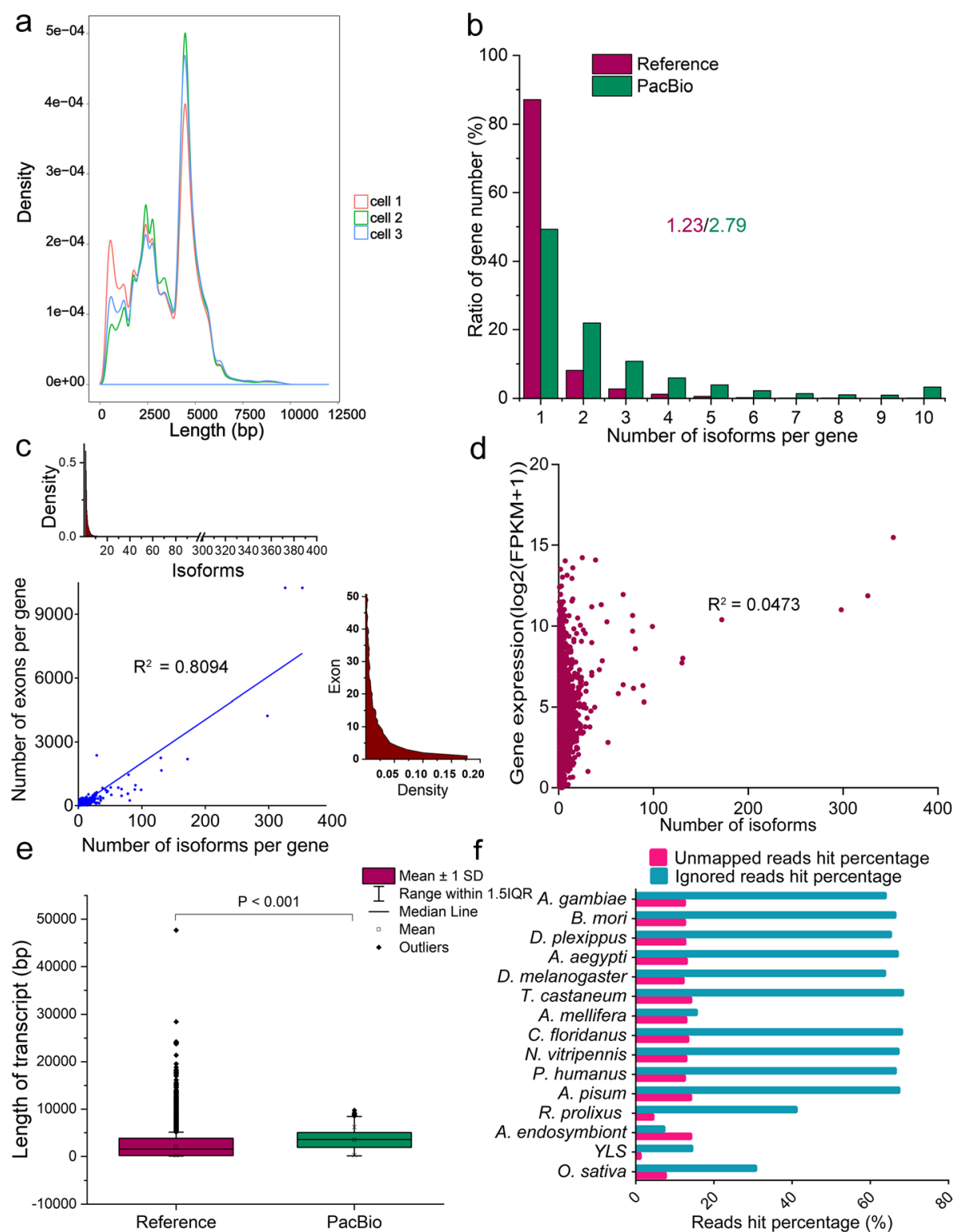

Fig. 1 Characteristics of transcript isoforms in N. lugens detected by PacBio sequencing. a, Distribution of insert reads' length in three SMRT cells. b. Comparison of number of isoforms per gene between transcripts in the reference genome NilLug1.0 and PacBio sequencing dataset. $\mathbf{c}_{\text {, }}$ Correlation between the number of isoforms per gene and number of exons per gene in the PacBio sequencing data (lower left graph), density profile of the number of exons per gene (right graph) and density distribution of the number of isoforms per gene (upper left graph). $\mathbf{d}$, Correlation between the number of isoforms per gene and gene expression level according to PacBio sequencing results. e, Comparison of length of transcripts in the reference genome NilLug1.0 and PacBio sequencing isoforms (with significance of difference determined by Wilcoxon rank sum test). f, Percentages of unmapped and filtered PacBio reads mapped to the genomes of 15 species using Blastx with an $\mathrm{E}<1 \mathrm{e}-5$

gene $\left(R^{2}=0.8094\right)$, but not expression level $\left(R^{2}=0.0473\right)$ (Fig. 1c, d). This is consistent with reported patterns in maize (Zea mays) [24]. By comparing the sequence structure of full-length transcripts with reference genome annotations, we identified 41,331 extension $\left(5^{\prime}\right.$ or $3^{\prime}$ end) in 5815 corresponding genes (Additional file 2: Table S1). The length of transcripts in the current study PacBio data (median, $3598 \mathrm{bp}$ ) is longer on average than that (median, $1561 \mathrm{bp}$ ) in the reference genome NilLug1.0 (Fig. 1e). The results clearly indicated that the full-length transcripts obtained by SMRT improved the annotation quality of the reference genome of $N$. lugens.

The unmapped reads $(1847 ; 1.06 \%$ of the total) and filtered PacBio reads $(29,073 ; 16.87 \%)$ were used as query sequences and further mapped to the genomes of 12 BPH-related species (Bombyx mori, Danaus plexippus, Anopheles gambiae, Aedes aegypti, Drosophila melanogaster, Tribolium castaneum, Apis mellifera, Camponotus 
Table 1 Data yield of single-molecule, real-time sequencing

\begin{tabular}{|c|c|c|c|c|}
\hline Sample & Cell 1 & Cell 2 & Cell 3 & All \\
\hline Number of reads of insert $(\mathrm{ROI})$ & 135,059 & 168,798 & 132,200 & 436,057 \\
\hline Mean length of ROI (bp) & 3258 & 3569 & 3533 & 3453 \\
\hline Mean number of passes & 12.25 & 10.61 & 11.06 & 11.31 \\
\hline $\begin{array}{l}\text { Number of five } \\
\text { prime reads }\end{array}$ & 126,260 & 158,466 & 122,763 & 407,489 \\
\hline $\begin{array}{l}\text { Number of three } \\
\text { prime reads }\end{array}$ & 126,962 & 157,275 & 123,642 & 407,879 \\
\hline Number of poly-A reads & 126,683 & 156,800 & 123,158 & 406,641 \\
\hline Number of full-length reads & 117,577 & 145,568 & 113,202 & 376,347 \\
\hline $\begin{array}{l}\text { Number of full-length } \\
\text { non-chimeric reads }\end{array}$ & 114,878 & 142,212 & 109,810 & 366,900 \\
\hline $\begin{array}{l}\text { Mean full-length non-chimeric } \\
\text { read length (bp) }\end{array}$ & 3088 & 3432 & 3367 & 3295 \\
\hline
\end{tabular}

floridanus, Nasonia vitripennis, Pediculus humanus, Rhodnius prolixus, and Acyrthosiphon pisum), two facultative microbial endosymbionts (yeast-like symbiont and Arsenophonus endosymbiont) [33] and the host rice $O$. sativa using Blastx with an $\mathrm{E}<1 \mathrm{e}-5$ cutoff (Additional file 3, Table S2). In addition to a few sequences with matches in host rice and endosymbiotic bacteria genomes, 'hits' were obtained for a number of the sequences in genomes of the BPH-related species (Fig. 1f). The results suggested a strong likelihood that these sequences are genuinely encoded in the BPH genome.

\section{Alternative splicing detection and verification}

In higher eukaryotic organisms, most genes are interrupted by introns. It is necessary to remove introns from the premessenger RNA accurately to form the mature messenger RNA. The intron boundary sequences at the $5^{\prime}$ and $3^{\prime}$ ends are recognized and processed by spliceosome component. Using a Perl script to analyze PacBio isoforms and reference transcripts, we detected 349 intron boundary sequence types in BPH genes. However, the highly conserved GT-AG type [34], accounted for $92.75 \%$ of the total number of detected boundaries (Fig. 2a; Additional file 4: Table S3).

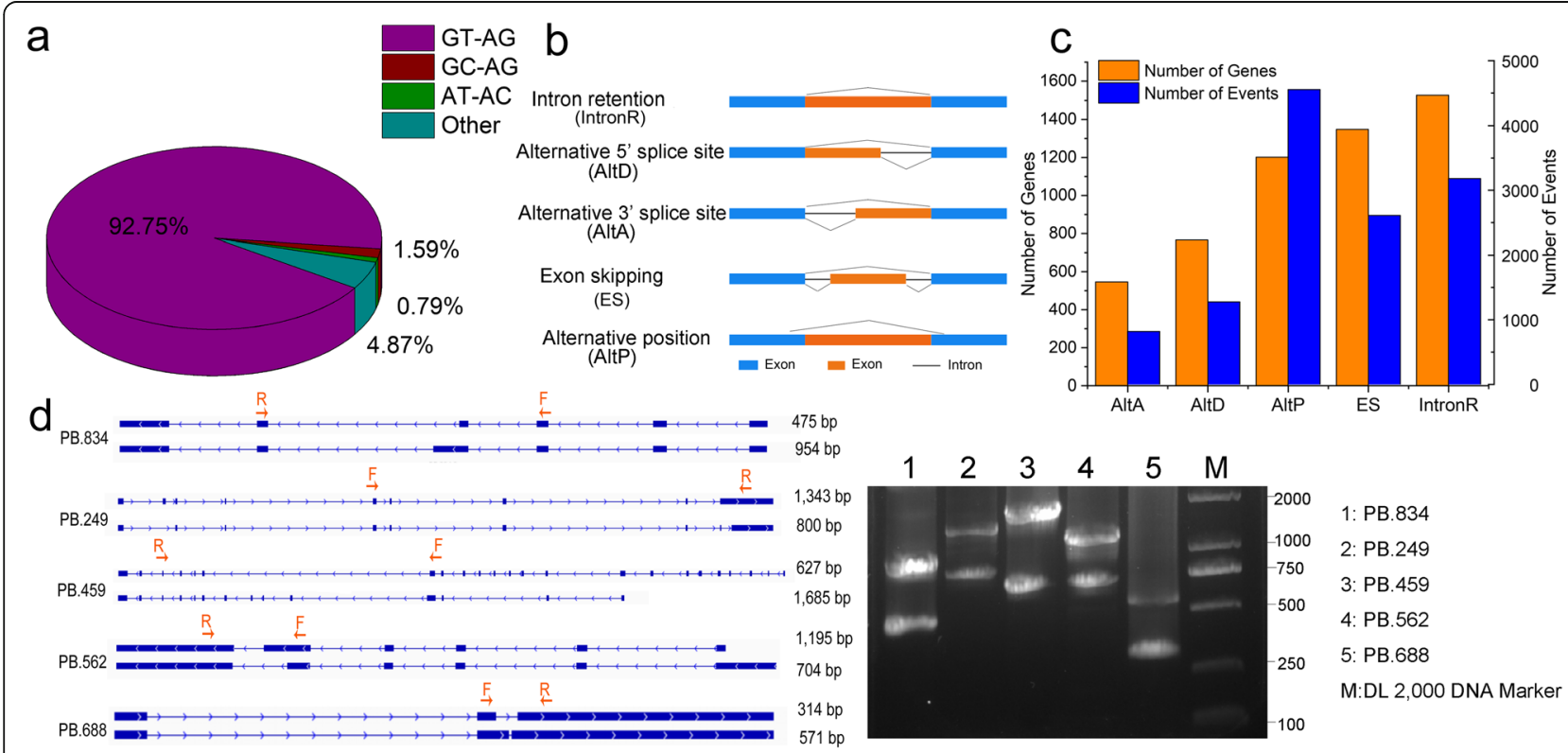

Fig. 2 Alternative splicing detection and verification in transcripts of N. lugens. a, Distribution of intron boundary types. b, Models for five types of alternative splicing. $\mathbf{c}$, Classification and statistics of alternative splicing events. $\mathbf{d}$, RT-PCR validation of AS events for five genes. The full-length transcript structure of each isoform is shown on the left. Blue boxes and blue lines represent exons and introns, respectively. Orientations from left to right and vice versa indicate that the transcript is located in positive and negative chains, respectively. PCR primers ( $F$, forward and $R$, reverse) are displayed on the first isoform of each gene. The length of PCR products is shown to the right of the structure of each transcript. Agarose gel electrophoresis bands in the right figure show the PCR result and DNA marker 
The multiplicity of splicing sites enables the generation of multiple isoforms of transcripts of the same gene through alternative splicing, thereby greatly extending the diversity of transcripts and proteins in eukaryotic organisms, and their regulatory complexity [35-37]. For example, previous analyses based on RNA-seq data have shown that transcripts of 31 and $25 \%$ of all genes in Drosophila melanogaster and Caenorhabditis elegans, respectively, are subject to alternative splicing [38, 39]. The current study PacBio full-length isoform data provide the first overall view of alternative splicing events in $\mathrm{BPH}$. The current study detected 20,356 alternative splicing events from 3594 genes, accounting for $18.15 \%$ of all protein-coding genes $(19,806)$ in BPH genome (Additional file 5: Table S4), and alternative splicing of 3119 genes' products was responsible for 5988 novel transcripts. These findings indicate that alternative splicing plays an important role in transcript diversity in $\mathrm{BPH}$. Using a custom Python script [40], five types of alternative splicing were identified: exon skipping (ES), alternative $3^{\prime}$ splice site (AltA), alternative $5^{\prime}$ splice site (AltD), intron retention (IntronR), and alternative position (AltP) (Fig. 2b). The current study found that genes with IntronR events accounted for the largest proportion, followed by genes with ES events. AltP was most common frequent occurrence type of alternative splicing in $\mathrm{BPH}$, followed by IntronR (Fig. 2c). By combined analysis of the proportion of genes affected and the number of occurrences in each AS type, the current study found that IntronR seems to be the major type of alternative splicing in $\mathrm{BPH}$, which is consistent with reported patterns in Drosophila melanogaster, Caenorhabditis elegans and Bombyx mori [38, 39, 41].

The current study randomly selected five genes and verified the accuracy of detected alternative splicing events by reverse transcription polymerase chain reaction (RTPCR) analysis and Sanger sequencing. The current study first analyzed sequences of full-length transcripts of these genes that would result from the alternative splicing events, then designed primers for RT-PCR amplification (Additional file 6: Table S5), and finally examined the products by agarose gel electrophoresis and Sanger sequencing. Both size of RT-PCR products and Sanger sequencing results matched transcripts detected by the single-molecule, real-time sequencing (Fig. 2d).

\section{Long non-coding RNA identification}

Long non-coding RNAs (lncRNAs) are non-protein-coding transcripts that are longer than $200 \mathrm{nt}$ and play important roles in gene expression regulation at various levels [42]. The BPH full-length isoforms that were aligned to the reference genome but lacked protein annotation were used to predict lncRNAs (2435 in total) (Additional file 7: Table S6). Of these predicted lncRNAs, 168 (median length, 1389 bp) matched annotated lncRNAs in the reference genome (which includes 1075 in total) (Additional file 7: Table S7), while 2267 (median length, 2172 bp) were novel (Fig. 3a; Additional file 7: Table S8). According to their positions in the genome, lncRNAs can be divided into six types: intergenic (lincRNA), intronic, sense, antisense, bidirectional and enhancer lncRNAs (Fig. 3b). The current study found that intergenic lncRNA $(1623,66.65 \%)$ was the most common type in BPH (Fig. 3c), in accordance with previous results of genome-wide lncRNA identification in $\mathrm{BPH}$ and Bombyx mori [43, 44]. Previously, 2470 lincRNAs were identified in Apis cerana, 1514 in A. mellifera and 1119 in D. melanogaster based on RNA-seq datasets $[45,46]$. The results indicate that lincRNA is the most frequent type in insects. LincRNAs reportedly regulate the expression of neighboring protein-coding genes, probably through participation in a cis-regulatory network [47-49]. The current study found that $19.14 \%$ of $N$. lugens lncRNAs overlap with, and $54.42 \%$ are located within $<10 \mathrm{~Kb}$ of known proteincoding genes (Fig. 3d). The result indicate that $N$. lugens lncRNAs tend to be close to protein-coding genes, and thus may speculatively participate in their cis-regulation.

Analysis of these predicted lncRNAs' structure showed that $77.91 \%$ (1897) had only one or two exons, almost five times as high as the corresponding proportion of proteincoding transcripts $(16.00 \%, 3592)$. Moreover, only $0.70 \%$ of the lncRNAs (17) has more than 10 exons, far less than the corresponding proportion $(39.68 \%, 8911)$ of proteincoding transcripts (Fig. 3e). The average lengths of the predicted lncRNAs and protein-coding transcripts are 2307 and 3565 bp, respectively (Fig. 3f). Expression profiling confirmed that lncRNAs are less highly expressed than protein-coding transcripts (Fig. 3g). Thus, the current study results clearly indicate that IncRNAs have fewer exons per transcript and lower expression levels than protein-coding transcripts in $\mathrm{BPH}$, in accordance with previous analyses of transcription patterns in both $\mathrm{BPH}$ and mammals $[43,50]$.

\section{Detection of novel genes and fusion transcripts}

A novel gene refers here to a gene putatively encoding a detected transcript that does not match any annotated gene in the BPH reference genome. From analysis of the current study PacBio data, we predicted 2664 coding sequences (CDS) from 1794 novel genes (Additional file 8: Table S9 and Table S10), with an average length of 819 bp (Fig. 4a). Among these 1794 novel genes, homologues of $1213(67.61 \%)$ were functionally annotated in at last one of the following databases: Non-redundant $(\mathrm{Nr})$, Swiss-Prot, Kyoto Encyclopedia of Genes and Genomics (KEGG), Gene Ontology (GO), RefSeq and Clusters of Orthologous Group (COG). Moreover, homologues of 77 genes were annotated in all six databases (Fig. 4b; Additional file 9). KEGG classification analysis showed 

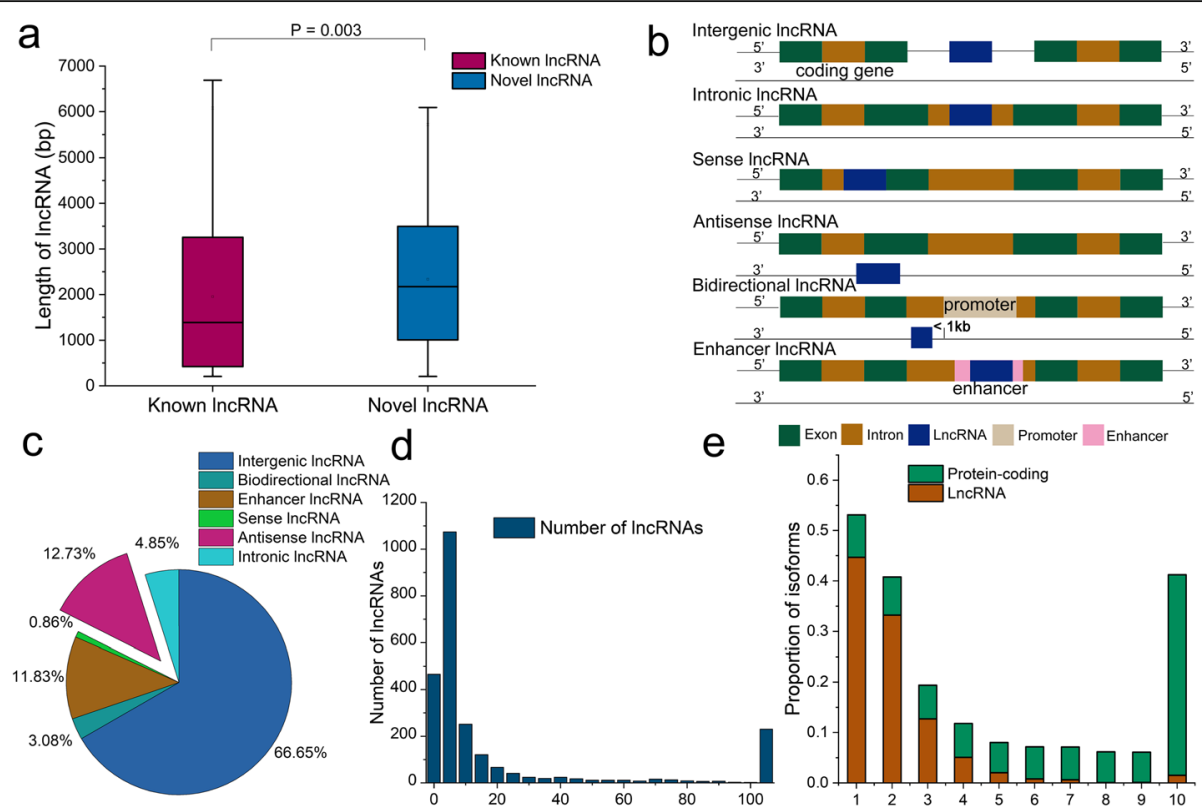

d

e
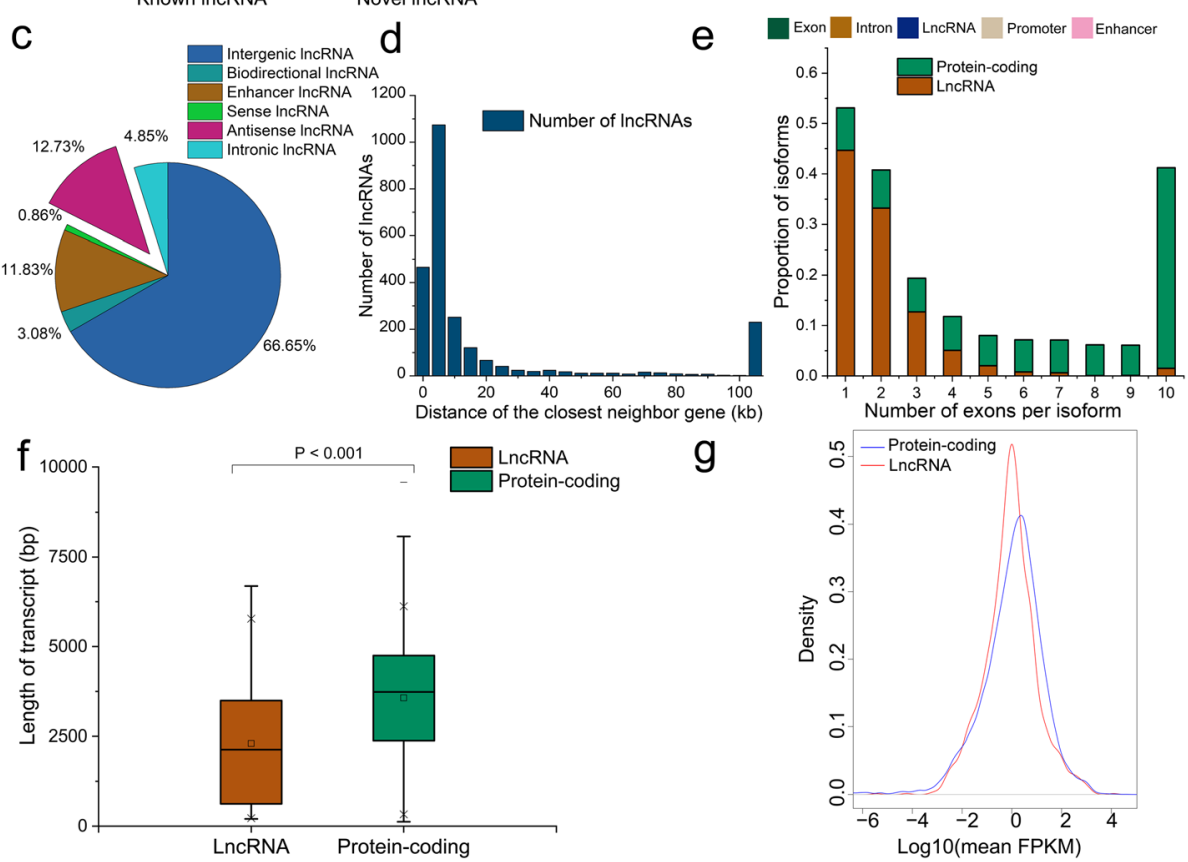

g

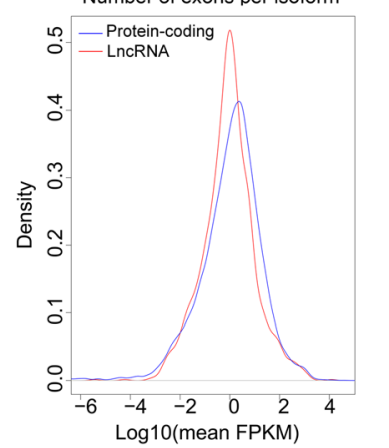

Fig. 3 Characteristics of IncRNAs in N. lugens. a, Comparison of transcript length between the novel IncRNAs and known IncRNAs (with significance of difference determined by Wilcoxon rank sum test). $\mathbf{b}$, Models for six types of IncRNA. c, Classification and statistics of IncRNA types. d, Frequency distribution of distances from the closest neighbor gene for IncRNA. e and $\mathbf{f}$, Comparison of the number of exons per transcript and transcript lengths between IncRNAs and protein-coding transcripts, respectively (with significance of difference in latter determined by Wilcoxon rank sum test). $\mathbf{g}$, Comparison of expression profiling between IncRNAs and protein-coding transcripts

that functions of the novel genes were mainly related to transport and catabolism, signal transduction, folding, sorting and degradation, carbohydrate metabolism, and endocrine system (Fig. 4c).

Fusion genes are formed by the cleavage and re-ligation of two different chromosomes, or cleavage and rearrangement of a chromosome, which binds normally separated transcriptional sequences [51]. Fusion genes are prevalent and common in cancer, and gene fusion is regarded as a distinct class of mutations [52], but there have been few analyses of gene fusion in insects. We detected 2631 reads supporting the presence of 915 fusion genes in the current study BPH samples (Additional file 10: Table S11). Of all the detected fusion genes, 417 (45.57\%) fused sequences of annotated genes at different locations in the reference genome, 105 (11.48\%) fused sequences of unannotated genes, and 393 (42.95\%) fused sequences of annotated and unannotated genes (Additional file 10: Table S12). Transsplicing links exons from two independent transcripts, generating chimeric RNA, which has been detected in most eukaryotes [53]. The fusion sites of different transcript are identical to their respective splice sites in the normal splicing process, which is the key indication of trans-splicing [24]. The current study found that fusion boundaries of 166 of the 417 transcripts representing fused sequences of annotated genes (27.82\%) corresponded to junctions in normal splicing (Additional file 11). This suggests that parts of fusion transcripts resulting from fusion of sequences of annotated genes may be generated by the RNAs trans-splicing machinery, which augments the BPH transcriptome's diversity and complexity. To further verify the fusion genes, nine fusion transcripts were randomly selected and validated by RT-PCR and Sanger sequencing analysis. Nine fusion transcripts were 

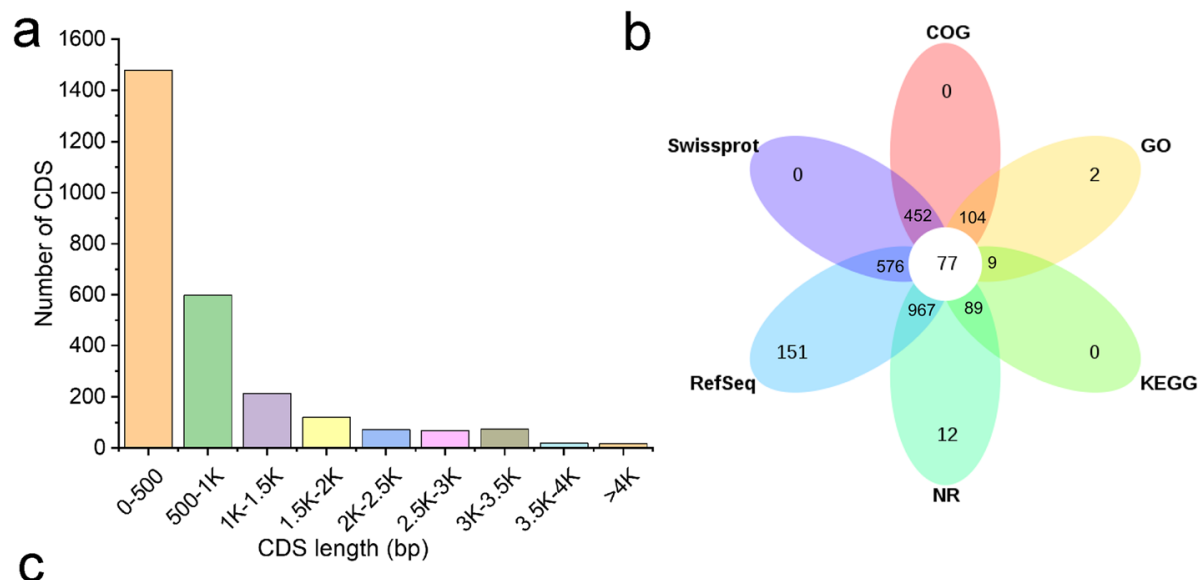

C

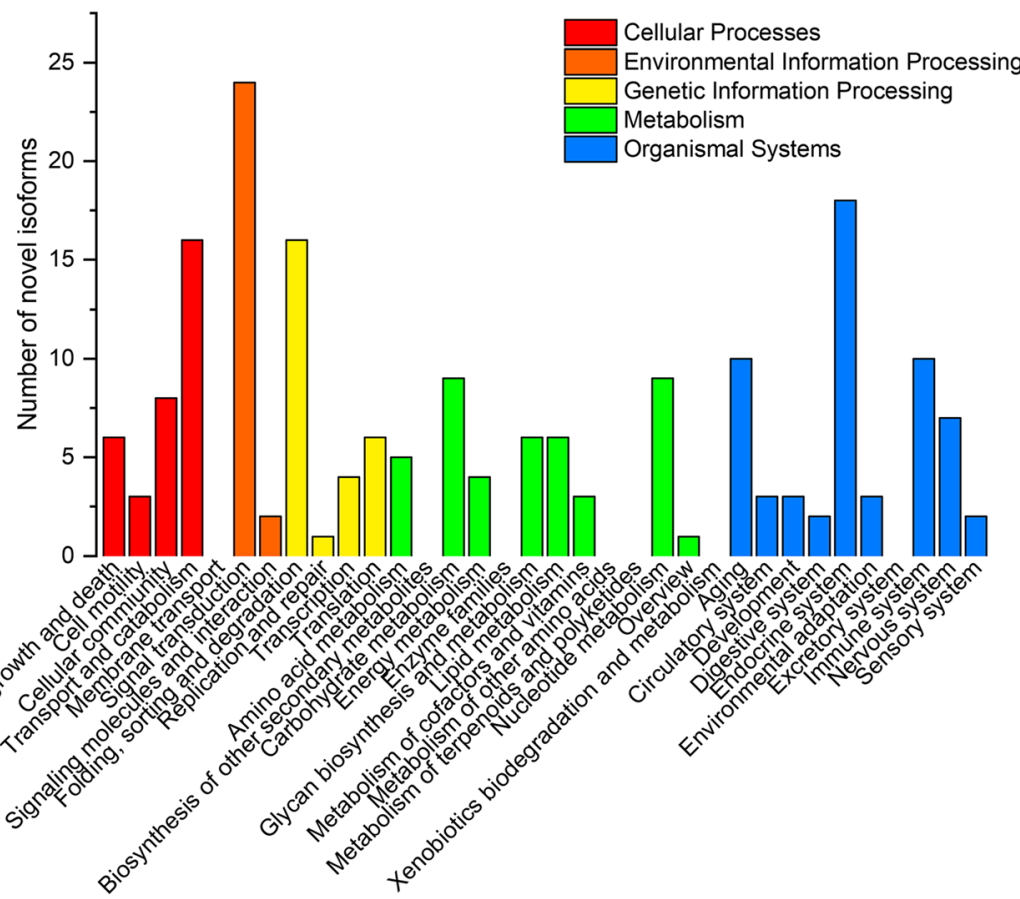

Fig. 4 Novel genes detected in N. lugens. a, CDS length distribution of novel genes. b. Ven diagram showing function annotations of novel genes in six databases. c, KEGG pathway classification of novel genes

experimentally validated, which confirmed the authenticity of these transcripts (Fig. 5a; Additional file 6: Table S5). GO classification analysis of all fusion genes revealed that functions of most of them were related to binding, catalytic activity, metabolic processes, and cellular processes (Fig. 5b).

\section{Differentially expressed genes in plant hoppers fed on resistant and susceptible rice plants}

The previous study has cloned the BPH-resistance gene $B p h 6$ in rice [16]. In this study, Bph6 was expressed under the control of native promoter and transformed into $\mathrm{BPH}$-susceptible Japonica rice variety Nipponbare by Agrobacterium tumefaciens-mediated transformation [54]. A T2 homozygous transgenic line (named Bph6- transgenic rice) with a single-copy insertion was used for $\mathrm{BPH}$ resistance evaluation (Additional file 12: Figure S2). The results indicated that Bph6-transgenic rice has high resistance to $\mathrm{BPH}$, whereas wild-type Nipponbare plants are susceptible and died after BPH infestation. The current study then observed the performance of newly emerged female $\mathrm{BPH}$ adults that were allowed to feed, for $48 \mathrm{~h}$, on both genotypes of rice plants, and found they had a significantly lower survival rate on Bph6transgenic rice (62.83\%) than on Nipponbare (95.11\%) (Additional file 13: Figure S3a). Two simply measurable indicators of $\mathrm{BPH}$ feeding activity, weight gain and honeydew extraction [55], were also significantly lower during feeding on Bph6-transgenic rice (0.29 and 8.81 $\mathrm{mg}$, respectively) than during feeding on Nipponbare 


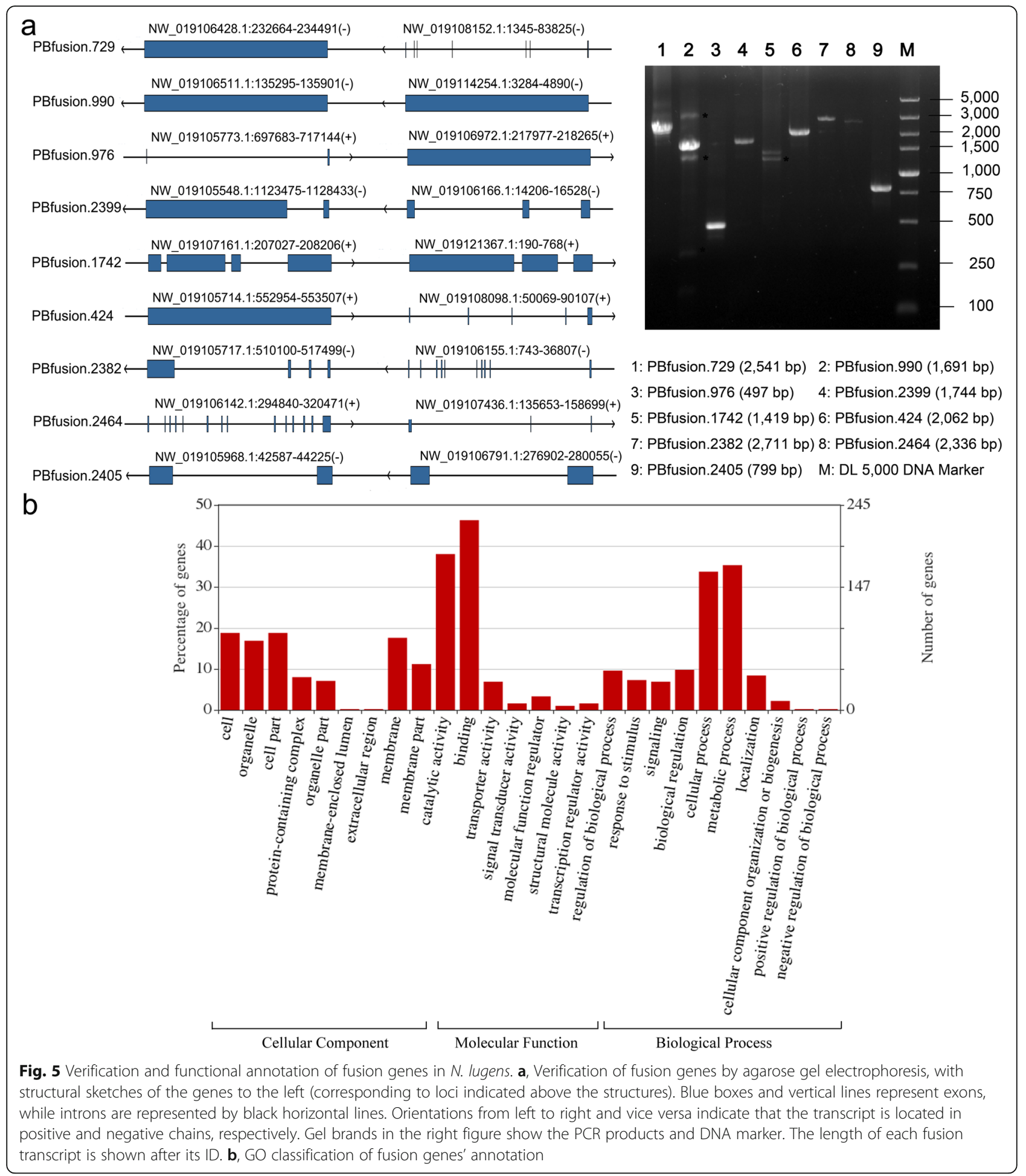

(1.17 and $62.15 \mathrm{mg}$, respectively) (Additional file 13: Figure S3b, S3c). Moreover, the weight gain ratio of $\mathrm{BPH}$ insects fed on Bph6-transgenic rice (0.14) was only about one-fifth of that on Nipponbare (0.76) (Additional file 13: Figure S3d). Thus, feeding on the Bph6-transgenic rice plants induced significant resistance stress in $\mathrm{BPH}$ insects, and we used them and wild type Nipponbare plants to characterize gene regulation in responses to host plant resistance in $\mathrm{BPH}$, via next generation RNAsequencing.

RNA samples from four biological replicates of insects fed on Nipponbare for $48 \mathrm{~h}$ (designated N-BPH) and 
others fed on Bph6-transgenic rice for $48 \mathrm{~h}$ (designated NB6-BPH) were sequenced using an Illumina HiSeq Xten PE150 platform (Table 2). Results of correlation analysis and Principal Component Analysis (PCA) showed that biological replicates correlated well (Additional file 14: Figure S4). Comparison of NB6-BPH and $\mathrm{N}-\mathrm{BPH}$ gene expression profiles identified 1893 differentially expressed genes (DEGs) with fold change (FC) $\geq 2$ and false discovery rate (FDR) $<0.01$ (Additional file 15: Figure S5; Additional file 16: Figure S6). Among these genes, 895 were up-regulated, and 998 were down-regulated in insects fed on Bph6-transgenic rice relative to those fed on Nipponbare (Additional file 17: Tables S13 and S14). The DEGs were subsequently subjected to GO and KEGG enrichment analyses based on Q values (adjusted $P$-values) $<0.05$ (Additional file 18: Tables S15 and S16; Additional file 19: Tables S17 and S18). They were classified into three main functional categories by GO enrichment analysis-molecular function (MF), cell composition (CC) and biological process (BP). As shown by the sets listed in Fig. 6a and b, the most strongly enriched GO terms in the up-regulated and down-regulated DEGs are associated with metabolic processes and catalytic activities, respectively.

KEGG pathway enrichment analysis (with a $Q$ value threshold of $<0.05$ ) showed that energy metabolism processes (including carbon metabolism, pentose phosphate pathway, glycolysis/gluconeogenesis, citrate cycle and fatty acid metabolism etc.) were the main up-regulated metabolic pathways. Moreover, up-regulated DEGs (but not down-regulated DEGs) were significantly enriched in the AMP-activated protein kinase (AMPK) signaling, FoxO signaling, longevity regulation and insulin signaling pathways (Fig. 6c). The down-regulated DEGs were mainly enriched in cell cycle and RNA transport. Moreover, genes involved in DNA replication, meiosis, and the digestion and absorption of fat and protein were also significantly enriched in down-regulated DEGs (Fig. 6d). These results clearly indicate that $\mathrm{BPH}$ mainly up-regulated metabolic processes and down-regulated cell proliferation at the transcriptional level in response to rice resistance stress.

Table 2 Statistics of RNA-seq data

\begin{tabular}{lllll}
\hline Sample & Read length (bp) & Clean reads & Clean bases & Q30 Rate (\%) \\
\hline N-1 & 150 & $39,212,486$ & $5,881,872,900$ & 89.87 \\
N-2 & 150 & $40,992,814$ & $6,148,922,100$ & 88.33 \\
N-3 & 150 & $44,493,422$ & $6,674,013,300$ & 89.11 \\
N-4 & 150 & $40,176,198$ & $6,026,429,700$ & 89.87 \\
NB6-1 & 150 & $40,234,308$ & $6,035,146,200$ & 89.55 \\
NB6-2 & 150 & $37,245,018$ & $5,586,752,700$ & 89.15 \\
NB6-3 & 150 & $40,161,428$ & $6,024,214,200$ & 89.92 \\
NB6-4 & 150 & $46,213,362$ & $6,932,004,300$ & 90.56 \\
\hline
\end{tabular}

To verify the reliability of the DEGs, 12 up-regulated genes and 15 down-regulated genes were randomly selected for real-time quantitative PCR (RT-qPCR), and the results were in good agreement with the RNA-seq data (Fig. 7; Additional file 6: Table S5).

\section{The FoxO signaling pathway is involved in $\mathrm{BPH}$ responses to resistance stress}

As mentioned above, genes associated with the AMPK signaling, FoxO signaling, longevity regulation, and insulin signaling pathways were significantly enriched in the upregulated DEGs in BPH insects fed on Bph6-transgenic rice. The FoxO signaling pathway is related to cell cycling, cell death, stress, detoxification, and other cellular processes [56]. FOXO transcription factors are regulated in response to nutrient deprivation by the AMPK pathway, and the AMPK-FOXO pathway plays a crucial role in the ability of a dietary restriction regimen to extend lifespan in C. elegans $[57,58]$. In addition, a FOXO ortholog is a crucial mediator of the insulin signaling pathway in Drosophila [59]. Therefore, the current study verified the expression of seven up-regulated genes (INRS, FOXO, caspase 8, ATG13, BNIP3, CAT and CAT-like) involved in the FoxO signaling pathway in NB6-BPH insects by RTqPCR (Fig. 8; Additional file 6, Table S5). The RT-qPCR results confirmed that the seven genes' expression was upregulated in all the $\mathrm{BPH}$ with inhibited weight gain on Bph6-transgenic rice (NB6-BPH), relative to N-BPH. It is well known that the FOXO transcription factor is activated and up-regulated under starvation conditions in insects $[60,61]$. Starvation also induces increases in expression of Nlbmm (another up-regulated DEG in NB6BPH: Fig. 8; Additional file 17: Table S13), while knockdown of Nlbmm confers significant hunger resistance in $\mathrm{BPH}$ [62]. These results all indicate that $\mathrm{BPH}$ responses to rice resistance might be related to starvation stress responses.

Caspase 8, ATG13 and BNIP3 are regulated by the FoxO signaling pathway and play important roles in apoptosis and autophagy [63-65]. These genes were significantly up-regulated, while IAP (which encodes an inhibitor of caspase [66, 67]), was significantly downregulated in NB6-BPH (Fig. 8). These results suggest that feeding on resistant Bph6-transgenic plants may induce cell apoptosis and autophagy in BPH. Furthermore, we found that the expression of genes associated to active oxygen elimination and detoxification, such as catalase (CAT), glutathione S-transferase (GST), methionine sulfoxide reductase (MSR), ferritin and carboxylesterases (CarE) [68] were significantly up-regulated in NB6-BPH relative to N-BPH (Fig. 9; Additional file 6, Table S5). These results indicated that BPH's adaptation to rice resistance stress may involve oxidative decomposition, and detoxification. 


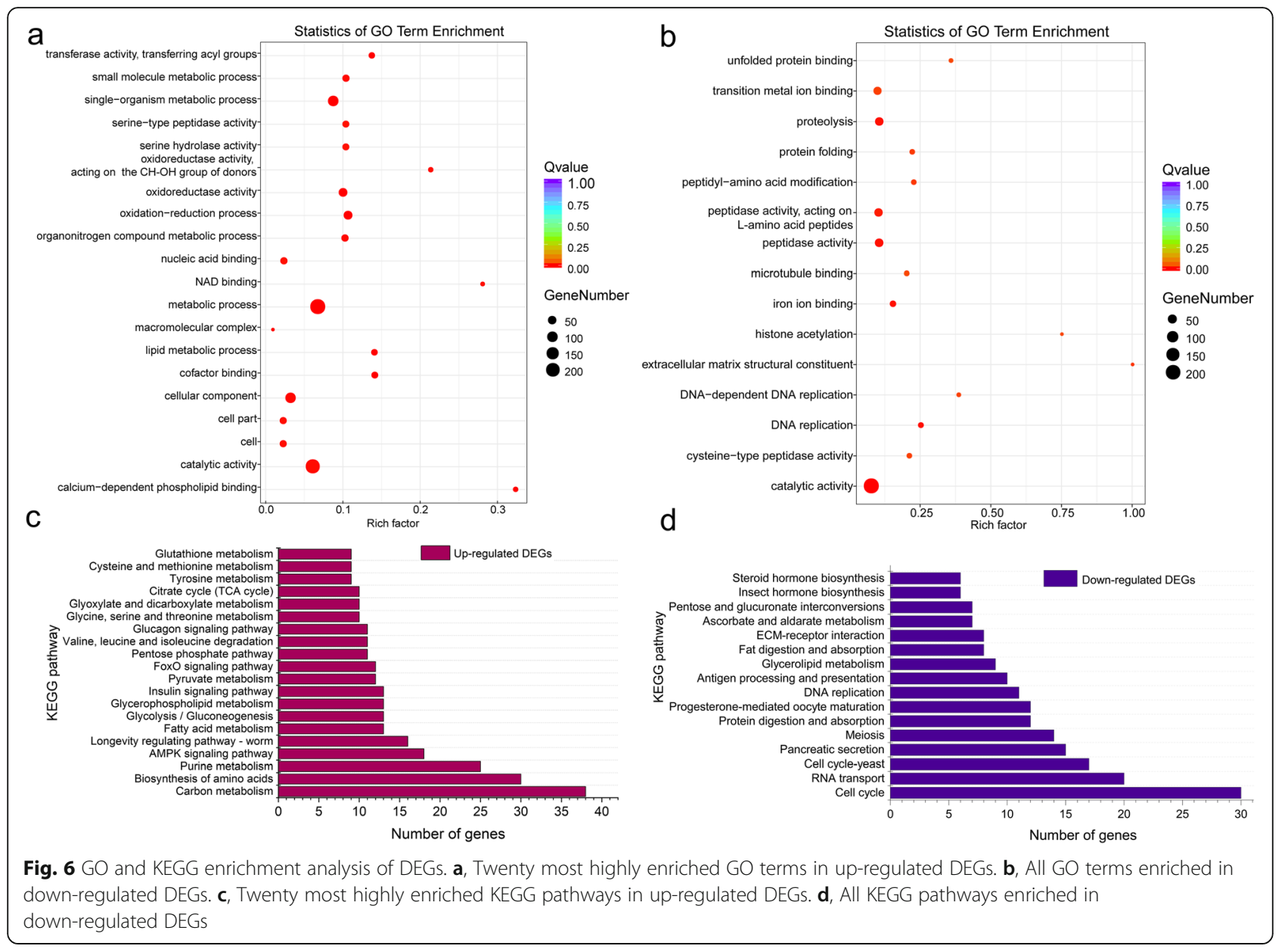

\section{Discussion}

The current study combined next-generation and singlemolecule sequencing to characterize the transcriptome of BPH biotype 1 and explore BPH responses to resistant rice plants. The results reveal the BPH transcriptome's diversity and complexity, and show that analysis of fulllength transcripts can improve annotation of the reference genome. The current study results also suggest that multiple pathways relating to starvation stress, active oxygen elimination and detoxification are regulated in $\mathrm{BPH}$ responses to resistant rice.

Nearly all of the single-molecule sequencing reads (98.94\%) were mapped to the BPH reference genome NilLug1.0, indicating the high quality of the sequences. Among 20,662 isoforms annotated to 7395 genes, most $(17,175 ; 83.12 \%)$ are novel transcripts that were not present in the reference genome database. The current study results illustrated the high diversity in the transcriptome of BPH that alternative splicing plays a crucial role in production of various $\mathrm{BPH}$ transcripts. The molecular diversity of insects' innate immune system is known to involve alternative splicing [69], but the current study single-molecule sequencing of the $\mathrm{BPH}$ transcriptome showed that fusion transcripts also contribute to its diversity. In total, it detected 1794 novel transcripts without annotations. Thus, the current study findings extend knowledge of both transcript diversity in $N$. lugens and the mechanisms involved.

The current study results also extend knowledge of lncRNAs in $\mathrm{BPH}$. In total, there are 1075 predicted lncRNAs in the BPH reference genome. However, the current study identified 2435 lncRNAs, of which 2267 were novel. In fruitfly and honeybee, lncRNA-regulated patterns include extensive trans-regulation by epiregulation of chromatin (re) organization or RNA sequestration in the nuclear compartment, and cis-regulation of neighboring protein-coding genes [70]. The current study found that intergenic lncRNA was the most common type in BPH. Whereas previous studies indicated that many intergenic lncRNAs are close to regulatory or developmental genes in the human, mouse and zebrafish genomes, and have potential cis-regulatory roles in protein-coding gene transcription [49]. The current study found that most of the BPH lncRNAs (1791; 73.56\%) overlap with or are located within $<10 \mathrm{~Kb}$ of known protein-coding genes, suggesting that they likely participate 


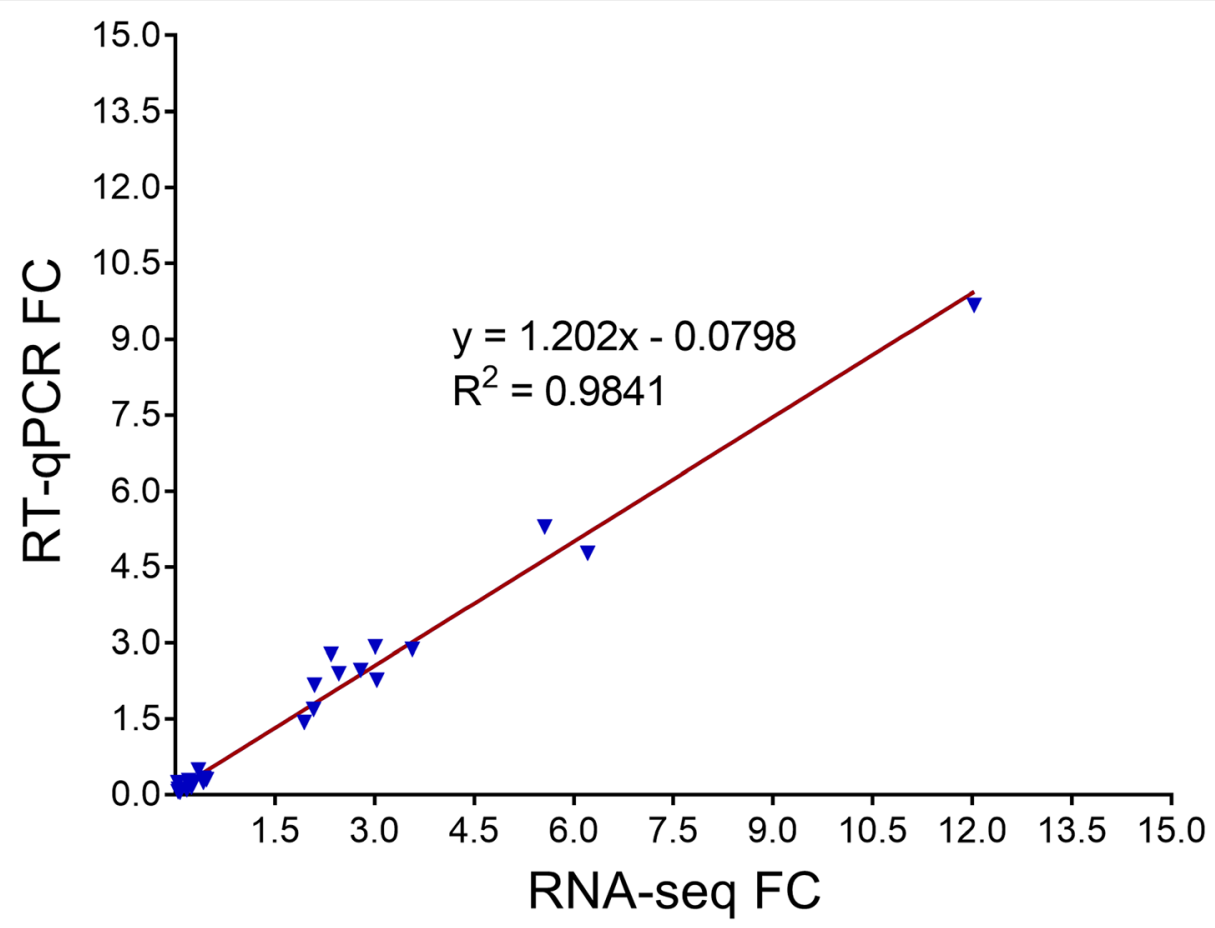

Fig. 7 Concordance of expression fold changes of genes detected in RNA-seq and RT-qPCR analyses. The blue triangles represent 12 upregulated genes and 15 down-regulated genes. BPH actin was used as an internal reference gene, three biological replicates were analyzed

in cis-regulation of these genes' transcription. These research findings add to the current study comprehension of the complexity of transcriptional regulation in $N$. lugens, also indicate that both expansion of transcriptional subtypes from multiple exon genes, without increases in the number of genes, and regulation of protein-coding genes by lncRNAs, may be involved in $\mathrm{BPH}$ 's adaptations to the wide variation in rice [71]. The diversity and complexity of BPH transcriptome may also be required for the insects to adapt to various changes in environmental conditions during long-distance migrations, which are crucial elements of their life history and reproduction cycles [72].

The $\mathrm{BPH}$ is a monophagous insect of rice. In the process of long-term interaction between $\mathrm{BPH}$ and rice, rice has evolved resistance to $\mathrm{BPH}$, which is controlled by the resistance genes. Previous studies have shown that during the long-term interactions between rice and its monophagous pest $\mathrm{BPH}$, rice has evolved genes that confer varying degrees of resistance to BPH through sieve tube sealing, and production of both secondary metabolites and various kinds of proteins [6-8]. These genes include Bph6, which encodes a novel protein that locates on exocyst and participates in regulation of phytohormone signal pathways and excretion in rice cells. Deposition of callose in sieve tubes, strengthening of cell walls and production of phytoalexin have been observed in Bph6 plants following infestation by $\mathrm{BPH}$ insects [16]. The current study verified that feeding on Bph6-transgenic rice plants reduced BPH insects' survival rate and feeding activity, relative to feeding on susceptible wild type plants. The current study also discovered that BPH up-regulated metabolic processes and down-regulated cell proliferation at the transcriptional level when feeding on Bph6-transgenic rice plants. These energy metabolism process including carbon metabolism, pentose phosphate pathway, glycolysis/gluconeogenesis, citrate cycle and fatty acid metabolism were significantly enriched in up-regulated DEGs. Energy mobilization processes are generally strengthened in insects during starvation, embryogenesis, and immune responses that enhance their survival chances when food is scarce [73]. In contrast, endoreplication only occurs if there is adequate nutrition, at least in D. melanogaster cell cultures [74]. Thus, the down-regulation of DEGs involved in cell proliferation observed when $\mathrm{BPH}$ fed on resistant rice may relate to nutrient deficiency resulted from resistant stress.

In response to attacks by herbivorous insects that minimize damage, plants defense against insects involve activation of both chemical and physical feeding deterrents that lead to insects starvation $[75,76]$. Presumably linked responses we detected in BPH responses to Bph6-transgenic rice plants included changes in expression of FoxO signaling pathway components. Overexpression of FOXO reportedly phenocopies starvation in $D$. melanogaster, suggesting that FOXO participates in adverse nutritional reactions [60,61]. Nlbmm (known to be up-regulated in $\mathrm{BPH}$ 

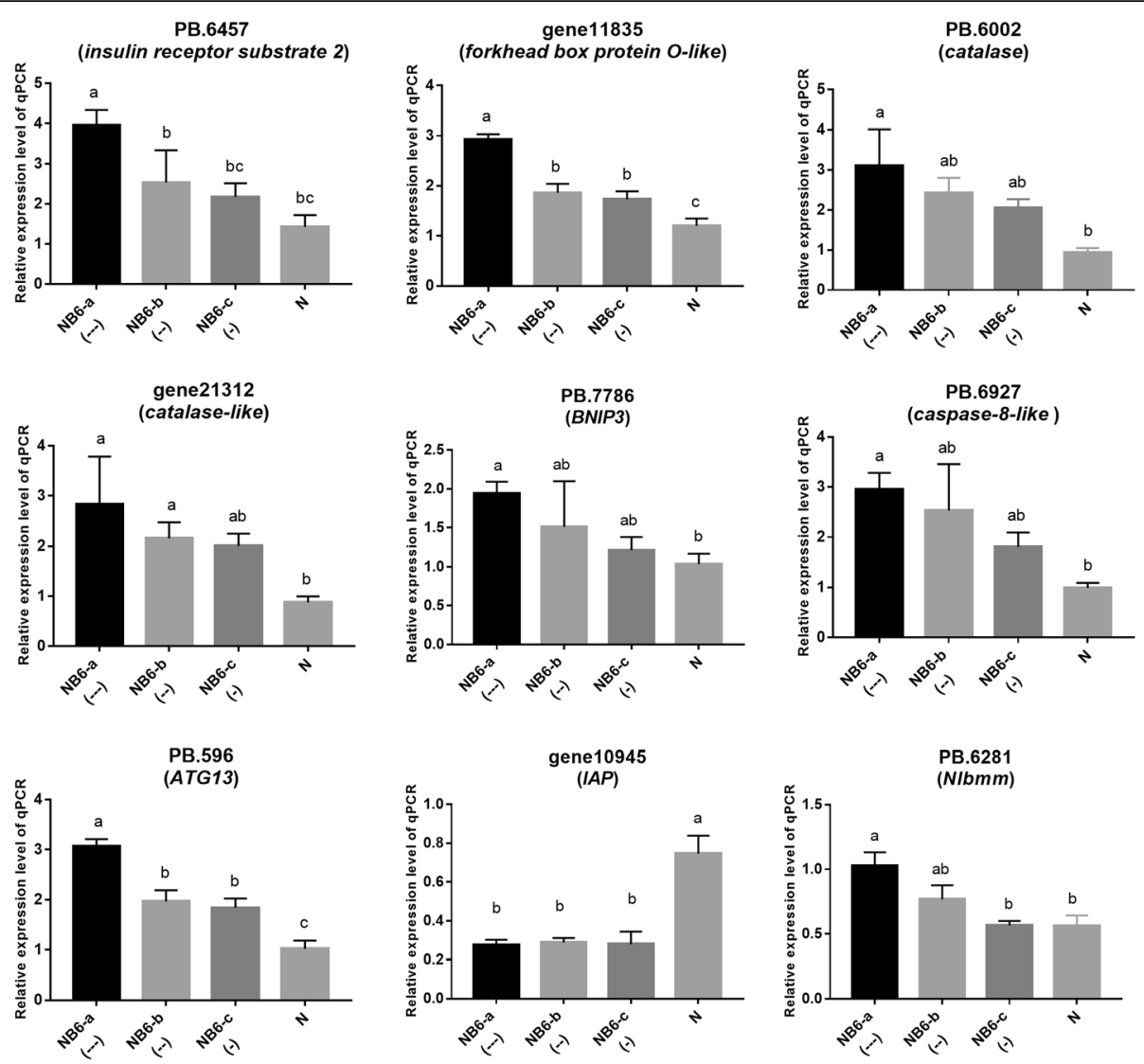

Fig. 8 Expression of nine genes in BPH insects fed on resistant and susceptible rice plants. The top seven genes were involved in the FoxO signaling pathway. The last two genes represent caspase inhibitor (IAP) and lipase brummer (N/bmm), respectively. N: BPH fed on Nipponbare susceptible rice for $48 \mathrm{~h}$, with $2.0 \mathrm{mg}$ weight gain. NB6-a(---), NB6-b(--) and NB6-c(-): BPH insects fed on Bph6-transgenic rice for $48 \mathrm{~h}$ with varying degrees of weight loss $(-0.4,-0.2$ and $-0.05 \mathrm{mg}$, respectively). Error bars represent SEM, $n=4$ independent experiments, different letters above the bars indicate significant differences, according to one-way ANOVA test $(P<0.05)$

starvation response [62]) was also up-regulated in NB6$\mathrm{BPH}$. These findings clearly indicate that some of the responses of BPH insects to short-term feeding on resistant rice plants are related to starvation stress. Other defenses of plants against insect herbivores include production of various secondary metabolites [77, 78]. However, one of the strategies for insects to overcome this problem is the detoxification of defense chemicals by oxidation, reduction, hydrolysis or conjugation of molecules [79, 80]. The current study found that genes related to active oxygen elimination and detoxification enzymes were up-regulated in $\mathrm{BPH}$ fed on Bph6-transgenic plants. Overall, the current study results imply that BPH responses to rice resistance stress include nutrient transformation, oxidative decomposition, and detoxification of xenobiotics.

\section{Conclusions}

The current study concluded that the first indications of the full diversity and complexity of BPH transcriptome at the isoform level and showed that analysis of the full-length transcripts can improve annotation of the reference genome. In addition, analysis of DEGs between $\mathrm{BPH}$ insects fed on Bph6-transgenic plants and others fed on susceptible wild type plants indicated that the BPH responses to host resistance stress might relate to starvation stress, nutrient transformation, active oxygen elimination and detoxification. These findings may facilitate further elucidation of interactions between insect herbivores and host plants.

There were several limitations also noticed like, first, the current study revealed characteristics of alternative splicing, fusion genes and lncRNAs in BPH at the isoform level, but did not further explore their functions in interactions with rice. Second, the previous studies shown that FOXO, PI3K/ Akt and MAPK pathways play significant roles in apoptotic responses of silkworm brain neurons to starvation [81], and starvation induced autophagic apoptosis in several insects [82-84]. Whereas, the current study found that genes related to starvation stress, apoptosis and autophagy were upregulated in BPH fed on Bph6-transgenic plant, but the possibility that feeding on resistant rice induces autophagic cell apoptosis in BPH insects warrants further study in continuation to current research findings. 


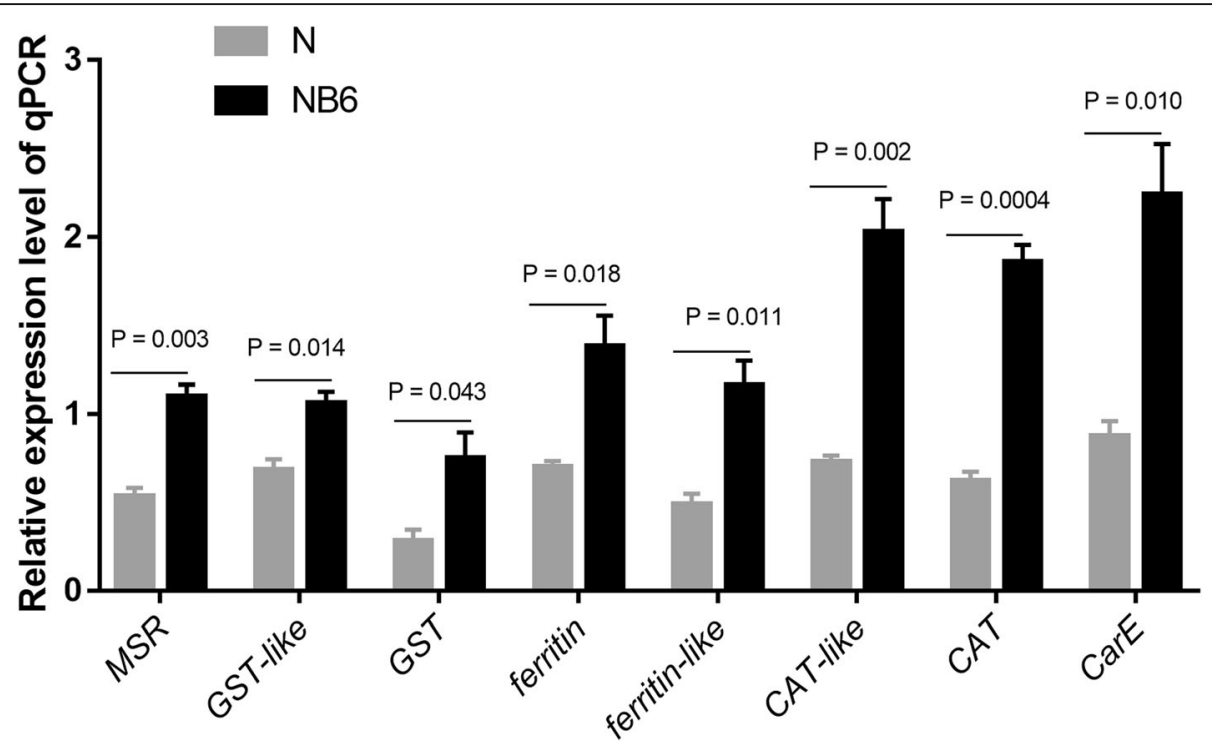

Fig. 9 Differentially expressed genes related to active oxygen elimination and detoxification between N-BPH and NB6-BPH. N: BPH fed on Nipponbare susceptible rice for $48 \mathrm{~h}$, with $2.0 \mathrm{mg}$ weight gain. NB6: BPH fed on Bph6-transgenic rice for $48 \mathrm{~h}$, with weight loss ( $0.5 \mathrm{mg}$ ). Error bars represent SEM, $n=3$, calculating $P$ Value with one-way ANOVA test

\section{Methods}

\section{Brown plant hopper insects}

BPH (Nilaparvata lugens) biotype 1 [85] insects were kept in the laboratory and maintained on 1-month-old plants of the susceptible rice cv Taichung Native1 (TN1) under controlled environmental conditions $\left(26 \pm 0.5^{\circ} \mathrm{C}\right.$, $16 \mathrm{~h} \mathrm{light} / 8 \mathrm{~h}$ dark cycles) at the Genetics Institute, Wuhan University [55]. Newly emerged BPH female adults (about 16-17 days old) were used in experiments.

\section{Source of seeds and generation of transgenic rice plants} Susceptible wild type Taichung Native1 (TN1, IRRI Acc. No. 00105) and Nipponbare (IRGC Acc. No. 136196) rice seeds used in this study, and the seeds of Bph6 carrying rice cultivar Swarnalata (IRRI Acc. No. 33964) were obtained from International Rice Research Institute.

The Bph6-transgenic line was maintained as previous protocol by Guo and coworkers [16]. The Bph6 genome sequence was amplified from Swarnalata genomic DNA with B6G-F and B6G-R primers (Additional file 6: Table S5) and cloned into the vector pCAMBIA1300, then transformed into the susceptible recipient line Nipponbare rice through the Agrobacterium-mediated method. Southern blot analysis was used to determine the number of insertion copies in transgenic $\mathrm{T}_{0}$ lines obtained. Rice genomic DNA was digested with the restriction enzymes DraI. Hybridization of nucleic acids with a 728 bp biotinylated $h p t$ II probe according to the operating manual of North2South Chemiluminescent Hybridization and Detection Kit (Thermo Scientific, Cat. No. 17097). Rice seedlings of the $T_{2}$ homozygous progeny in the four-leaf stage from an independent Bph6-transgenic line were infected by second to third instar $\mathrm{BPH}$ nymphs for resistance evaluation, using the susceptible recipient line Nipponbare as a control. $T_{2}$ homozygous progeny were identified by PCR analysis of their genomic DNA, using the Bph6-specific B6O-F and B6O-R primers (Additional file 6: Table S5), and the constructed vector and Nipponbare DNA as a positive and negative controls, respectively. A voucher specimen of the Bph6-transgenic line has been deposited in the China Center for Type Culture Collection (CCTCC No. P201907).

\section{Sample collection and RNA preparation}

Susceptible wild type Nipponbare rice and Bph6-transgenic rice were used in BPH feeding experiments, as follows. Seeds of both types were sown in plastic cups (radius $4 \mathrm{~cm}$, height $20 \mathrm{~cm}$ ), five per cup, then cultivated in a greenhouse providing $30 \pm 2{ }^{\circ} \mathrm{C} / 14 \mathrm{~h}$ light and $28 \pm$ $2{ }^{\circ} \mathrm{C} / 10 \mathrm{~h}$ dark cycles at Wuhan University $\left(30^{\circ}\right.$ north latitude and $114^{\circ}$ eastern longitude). When rice plants of both types had grown from the seeds to the five-leaf stage (about 4 weeks after sowing) the performance of adult female $\mathrm{BPH}$ was measured [86]. The research evaluation involved data observation on $\mathrm{BPH}$ adult female with main traits viz. survival rate, weight gain, honeydew excretion, and weight gain ratio after feeding for $48 \mathrm{~h}$ on the plants.

In addition, 80 insects fed on Nipponbare for $48 \mathrm{~h}(\mathrm{~N}$ $\mathrm{BPH}$ ) [87] were randomly sampled in sets of four biological replicates, with 20 insects per replicate $[88,89]$. Similarly, 80 insects fed on Bph6-transgenic rice for $48 \mathrm{~h}$ with the inhibited weight gain (NB6-BPH) were sampled for four biological replicates. As already mentioned the 
antenna, stylet, salivary gland and leg play important roles in BPH's exploration and acquisition of food from rice plants $[28,29]$. Therefore, the current study dissected heads and forelegs of BPH female insect, which contain these organs, from the sampled insects under a stereo microscope on ice. Tissues of the 20 insects in each group were mixed and total RNA was extracted from them, using an EASYspin tissue/cell RNA rapid extraction kit (Cat. \#HF107-02). The Agilent 2100 Bioanalyzer (Agilent Technologies, USA) and agarose gel electrophoresis system (Lonza, Switzerland) were used to confirm the integrity of all RNA samples, and a NanoDrop 2000c Spectrophotometer (Thermo Scientific, USA) was used to measure RNA concentration.

\section{Illumina library construction and RNA sequencing}

Sequencing libraries were constructed from high-quality RNA samples (OD260/280 $=1.8 \sim 2.2$, OD260/230 $\geq 2.0$, RIN $\geq 8$, RNA $>1 \mu \mathrm{g}$ ) obtained from the replicates using a TruSeq ${ }^{\circ}$ RNA Sample Preparation Kit v2 (Illumina, USA). PolyA mRNA was purified from total RNA $(1.5 \mu \mathrm{g}$ per sample), then fragmented. First-strand cDNA was synthesized using reverse transcriptase, random primers and these short fragments as templates, further the second-strand cDNA was synthesized. The resulting synthetic cDNA was end-repaired and adenylated, then Illumina Adaptors were ligated to prepare for hybridization. After that, the library fragments were purified and PCR amplification were performed following the standard library construction protocol [90]. At last, the library preparations were subjected to pairedend sequencing using an Illumina HiSeq $\mathrm{X}$ Ten platform.

\section{Library preparation and PacBio sequencing}

Equal portions of the four total RNA samples obtained from NB6-BPH insects were pooled to construct a mixed RNA sample for PacBio sequencing. A SMARTer ${ }^{\text {tw }}$ PCR cDNA Synthesis Kit (Takara, Cat. Nos. 634,925 and 634, 926) was used for reverse transcription of the qualified RNA to cDNA, which was then subjected to PCR amplification using PrimeSTAR ${ }^{\circ}$ GXL DNA polymerase (Takara, R050A) with optimized (12) cycles. Amplified products of suitable sizes (0.5-6 Kb fragments) were then amplified by large-scale PCR to obtain enough DNA, and a SMRTbell Template Prep Kit (Pacific Biosciences, 101-357-000) was used to construct a SMRTbell library. Sequel libraries with insertion fragments of 1-6 Kb were constructed and sequenced using a PacBio Sequel platform with V2.1 chemistry (Pacific Biosciences) and $10 \mathrm{~h}$ movies.

\section{Pipeline for PacBio data analysis}

The quality of the sequencing reads was controlled by filtering out invalid reads and sequences with full pass less than
1 or accuracy less than 0.8 , to obtain high quality insert reads. These reads were then divided into full-length, nonfull-length and chimeric reads using the SMRT analysis software provided by Pacific Biosciences (http://www.pacb. com/products-and-services/analytical-software/smrt-analysis/). Subsequently, full-length non-chimeric (FLNC) reads were clustered and corrected by the Iterative Clustering for Error Correction (ICE) algorithm [30]. Lordec [31] was then applied to correct the clustering results with the RNA-seq data. All full-length reads were aligned with the BPH reference genome NilLug1.0 by GMAP [32] software, then collapsed by a Python script (collapse_isoforms_by_ sam.py), with 0.9 minimum identity and 0.85 minimum coverage. MatchAnnot software (https://github.com/TomSkelly/MatchAnnot) was used to compare the alignment results with NilLug1.0 annotation.

\section{Identification of alternative splicing}

Alternative splicing was identified and classified, using a Python script (https://github.com/Nextomics/pipeline-forisoseq) [40], into five types: exon skipping (ES), alternative $3^{\prime}$ splice site (AltA), alternative $5^{\prime}$ splice site (AltD), intron retention (IntronR), and alternative position (AltP).

\section{Prediction of IncRNA and novel genes}

An online tool (https://bitbucket.org/arrigonialberto/ Incrnas-pipeline) was used to identify putative lncRNAs in the PacBio dataset that aligned with the reference genome but not annotated transcripts. The predicted lncRNAs were then compared with known lncRNAs in reference genome NilLug1.0. Those transcripts which could not be aligned to gene models in NilLug1.0 and not be predicted to be lncRNA were considered as novel transcripts.

\section{CDS prediction and functional annotation}

The CDS and proteins encoded by all novel genes were predicted using ANGEL (https://github.com/PacificBiosciences/ANGEL) [91]. They were then functionally annotated by Blastx searches gainst the Nr, COG, Swiss-Prot, GO, RefSeq and KEGG databases with the cut-off e-value $\leq 1 \mathrm{e}-5$ and highest score criteria.

\section{Identification of fusion transcripts in PacBio sequences}

Fusion transcripts were identified using a Python script (fusion_finder.py) in the PBTRANSCRIPT-TOFU package (http://github.com/PacificBiosciences/cDNA_primer/) [40], with the following criteria: (a) mapping of a FLNC transcript to two or more annotated loci in the genome; (b) at least $10 \%$ of the transcript aligning with each mapped locus; (c) at least 99\% combined alignment coverage; (d) at least $10 \mathrm{~kb}$ distance between each mapped locus; and (e) a certain amount of Illumina reads supporting the fusion regions [24]. 


\section{Differential expression gene analysis}

Mapped reads of all genes were subjected to differential expression analysis using the edge $\mathrm{R}$ package [92]. Gene expression levels were calculated in terms of reads per kilobase of transcript sequence per million base pairs sequenced (FPKM) using stringTie [93]. The P.adjust function was used to adjust the generated $P$-value to control the false discovery rate. The threshold values for DEG significance were $P$-value $\leq 0.05$ and absolute value $\log _{2}$ (group1/group2) $\geq 1$. The DEGs were clustered based on FPKM by the gplots package (http://cran.r-project.org/ web/packages/gplots/index.html), then functionally annotated by BlastX searches (e-value $\leq 1 \mathrm{e}-5$ ) against the $\mathrm{Nr}$, COG, Swiss-Prot, GO, RefSeq and KEGG databases. The online tool provided at https://sourceforge.net/p/enrichmentpipeline/wiki/Home/ was used for $\mathrm{GO}$ term and KEGG pathway enrichment analysis.

\section{Reverse transcription PCR (RT-PCR)}

The RNA samples used for sequencing were also subjected to reverse transcription polymerase chain reaction (RT-PCR) analysis, with primers designed using primer-BLAST (https://www.ncbi.nlm.nih.gov/tools/primerblast/). The PCR products were isolated and cloned into the PMD18-T Vector (Takara, Cat. \#6011) for Sanger sequencing to validate PacBio isoforms.

\section{Real-time quantitative PCR (RT-qPCR)}

cDNA was synthesized from $2 \mu \mathrm{g}$ portions of total RNA using a PrimeScript RT reagent Kit with gDNA Eraser (TaKaRa, Cat. \#RR047A), and SYBR green Supermix (BioRad, Cat. \#172-5274) was used to amplify cDNA. Real-time quantitative PCR was performed with a CFX96 real-time system, following the manufacturer's instructions to verify the RNA-Seq analysis results. Sequences of primers used in real-time RT-PCR are listed in Additional file 6. BPH actin was used as an internal reference gene. At least three biological replicates were used in each experiment.

\section{Additional files}

Additional file 1: Figure S1. Workflow of combined SMRT and RNA-seq analysis of BPH. (TIF $2045 \mathrm{~kb}$ )

Additional file 2: Table S1. Optimization of sequences' structure. (XLSX $1879 \mathrm{~kb})$

Additional file 3: Table S2. Hits for unmapped and filtered PacBio reads in 15 species' genomes with an $\mathrm{E}<1 \mathrm{e}-5$. (DOCX $15 \mathrm{~kb}$ )

Additional file 4: Table S3. Intron boundary sequences at $5^{\prime}$ and $3^{\prime}$ ends in the BPH genome. (XLSX $15 \mathrm{~kb}$ )

Additional file 5: Table S4. Alternative splicing events detected in female adults of BPH biotype 1. (XLSX $1015 \mathrm{~kb}$ )

Additional file 6: Table S5. All primers used in the study. (DOCX $22 \mathrm{~kb}$ )

Additional file 7: Long non-coding RNA identification. Tables S6-S8: All 2435 predicted long non-coding RNA sequences, 168 known long non-coding RNA sequences, and 2267 novel long non-coding RNA sequences. (XLSX $3777 \mathrm{~kb}$ )

Additional file 8: Detection and CDS of novel genes. Tables S9. and S10: All 1794 predicted novel gene sequences and 2664 CDS sequences for these predicted novel genes, respectively. (XLSX $3266 \mathrm{~kb}$ )

Additional file 9: Functional annotations of novel genes in six databases: Nr, Swiss-Prot, KEGG, GO, RefSeq and COG. (XLSX 382 kb)

Additional file 10: Detection of fusion genes. Table S11. All detected fusion genes' sequences. Table S12. Positions of 915 fusion genes. (XLSX $1247 \mathrm{~kb})$

Additional file 11: Boundary sites of transcripts resulting from fusion of sequences of annotated genes at different locations. (XLSX $44 \mathrm{~kb}$ )

Additional file 12: Figure S2. Southern blotting analysis and resistance evaluation of $T_{2}$ progeny of Bph6-transgenic rice plants. A, Southern blotting analysis of $\mathrm{T}_{0}$ progeny of Bph6-transgenic rice plants. The sample in the first lane indicated by the arrow is from a T0 line with a single-copy insertion, and the T2 homozygous genetic line used in this study originated from this line. B, Photograph of susceptible recipient rice Nipponbare and the $T_{2}$ homozygous progeny of Bph6-transgenic rice after 5 days under BPH infestation. C, Amplification of Bph6 cDNA sequences in constructed vector plasmid, Bph6-transgenic and Nipponbare rice plants. (TIF $5761 \mathrm{~kb}$ )

Additional file 13: Figure S3. Performance of $\mathrm{BPH}$ insects on susceptible and resistant rice plants. a-d, Survival rate, weight gain, honeydew excretion and weight gain ratio of BPH female adults fed on Nipponbare and Bph6-transgenic plants. Error bars represent SEM, $n=3$ independent experiments, $P$ values were derived from one-way ANOVA test. (TIF $617 \mathrm{~kb}$ )

Additional file 14: Figure. S4. Results of correlation analysis and Principal Component Analysis (PCA) of all RNA samples. (TIF 3096 kb)

Additional file 15: Figure S5. Volcano map of differentially expressed genes between N-BPH and NB6-BPH. Green dots represent significant differentially expressed genes; red dots represent non-significant differentially expressed genes. The significant difference criteria were fold change $(F C) \geq 2$ and false discovery rate (FDR) $<0.01$. (TIF $1124 \mathrm{~kb}$ )

Additional file 16: Figure S6. Hierarchical clustering analysis of 1893 DEGs based on the log ratio of FPKM. The color key represents FPKMnormalized $\log _{2}$ transformed counts by Z-score standardization. Red and green indicate up-regulated and down-regulated, respectively. Each column shows a sample and each row represents a gene. (TIF 178 kb)

Additional file 17: All 1893 DEGs between N-BPH and NB6-BPH. Table S13. DEGs up-regulated in NB6-BPH relative to N-BPH. Table S14. DEGs down-regulated in NB6-BPH relative to N-BPH. (XLSX 265 kb)

Additional file 18: $\mathrm{GO}$ enrichment of DEGs between N-BPH and NB6$\mathrm{BPH}$. Table S15. GO enrichment of DEGs up-regulated in NB6-BPH relative to N-BPH. Table S16. GO enrichment of DEGs down-regulated in $\mathrm{NB6}-\mathrm{BPH}$ relative to $\mathrm{N}-\mathrm{BPH}$. (XLSX $27 \mathrm{~kb}$ )

Additional file 19: KEGG enrichment of DEGs between N-BPH and NB6$\mathrm{BPH}$. Table S17. KEGG enrichment of DEGs up-regulated in NB6-BPH relative to N-BPH. Table S18. KEGG enrichment of DEGs down-regulated in $\mathrm{NB6}-\mathrm{BPH}$ relative to $\mathrm{N}-\mathrm{BPH}$. (XLSX $16 \mathrm{~kb})$

\section{Abbreviations}

AltA: Alternative 3' splice site; AltD: Alternative 5' splice site; AltP: Alternative position; AMPK: AMP activated protein kinase; AS: Alternative splicing; ATG13: Autophagy-related protein 13; BNIP3: Bcl2/adenovirus EIB 19kDinteracting protein 3; BP: Biological process; BPH: Brown plant hopper; CarE: Carboxylesterases; CAT: Catalase; CC: Cell composition; CDS: Coding sequence; COG: Clusters of Orthologous Group; DEG: Differentially expressed gene; ES: Exon skipping; FC: Fold change; FDR: False discovery rate; FL: Fulllength; FLNC: Full-length non-chimeric; GMAP: Genomic Mapping and Alignment Program; GO: Gene Ontology; GST: Glutathione S-transferase; ICE: Iterative Clustering for Error Correction; INRS: Insulin receptor substrate; IntronR: Intron retention; KEGG: Kyoto Encyclopedia of Genes and Genomics; lincRNAs: Long intergenic non-coding RNA ; IncRNA: Long non-coding RNA; LoRDEC: Long Read DBG Error Correction; MF: Molecular function; 
MSR: Methionine sulfoxide reductase; NB6-BPH: BPH biotype 1 fed on Bph6transgenic rice plants; N-BPH: BPH biotype 1 fed on susceptible rice Nipponbare plants; NGS: Next-generation sequencing; Nlbmm: Nilaparvata lugens brummer; Nr: Non-redundant; PCA: Principal Component Analysis; RNA-Seq: RNA sequencing; ROI: Read of insert; RT-PCR: Reverse transcription polymerase chain reaction; RT-qPCR: Real-time quantitative polymerase chain reaction; SMRT: Single-molecule, real-time

\section{Acknowledgements}

Not applicable.

\section{Authors' contributions}

GCH conceived the project and research plans; JZ carried out most of the experiments, analyzed the data and wrote most of the article; $\mathrm{WG}, \mathrm{CMH}$, $Y X H, Y C, J P G, C Z, R Z C, B D, L L Z$ and DH carried out some of the experiments; $\mathrm{GCH}$ supervised and participated in the writing. All authors read and approved the final manuscript.

\section{Funding}

This work was supported by the National Natural Science Foundation of China (grant nos. 31230060 and 31171525). The funding agencies played no role in the design of the study, data collection, analysis, and interpretation or writing the manuscript.

\section{Availability of data and materials}

The PacBio SMRT reads and Illumina RNA-seq reads supporting conclusions presented in this article are available in the NCBI Sequence Read Archive (SRA). The accession number for the SMRT reads is SRR8442724 under BioProject PRJNA514182. Accession numbers for the Illumina cDNA libraries obtained from the BPH biotype 1 fed on the susceptible and Bph6-transgenic rice plants for $48 \mathrm{~h}$ are SRR8437756, SRR8437757, SRR8437758, SRR8437759, SRR8437760, SRR8437761, SRR8437762 and SRR8437763 under BioProject PRJNA514182.

\section{Ethics approval and consent to participate}

Not applicable.

\section{Consent for publication}

Not applicable.

\section{Competing interests}

The authors declare that they have no competing interests.

Received: 30 April 2019 Accepted: 20 August 2019

Published online: 29 August 2019

\section{References}

1. War AR, Paulraj MG, Ahmad T, Buhroo AA, Hussain B, Ignacimuthu S, et al. Mechanisms of plant defense against insect herbivores. Plant Signal Behav. 2012;7:1306-20

2. Kessler A, Baldwin IT, PLANT RESPONSES TO INSECT HERBIVORY. The emerging molecular analysis. Annu Rev Plant Biol. 2002;53:299-328.

3. Will T, Furch AC, Zimmermann MR. How phloem-feeding insects face the challenge of phloem-located defenses. Front Plant Sci. 2013;4:336.

4. Giordanengo P, Brunissen L, Rusterucci C, Vincent C, Van Bel A, Dinant S, et al. Compatible plant-aphid interactions: how aphids manipulate plant responses. C R Biol. 2010;333:516-23.

5. Zhao Y, Huang J, Wang Z, Jing S, Wang Y, Ouyang Y, et al. Allelic diversity in an NLR gene BPH9 enables rice to combat planthopper variation. Proc Natl Acad Sci. 2016;113:12850-5.

6. Hao P, Liu C, Wang Y, Chen R, Tang M, Du B, et al. Herbivore-induced callose deposition on the sieve plates of rice: an important mechanism for host resistance. Plant Physiol. 2008;146:1810-20.

7. Cheng $X$, Zhu L, He G. Towards understanding of molecular interactions between Rice and the Brown Planthopper. Mol Plant. 2013;6:621-34

8. Maffei ME, Mithöfer A, Boland W. Before gene expression: early events in plant-insect interaction. Trends Plant Sci. 2007:12:310-6.

9. Cabauatan PQ, Cabunagan RC, Choi IR. Rice viruses transmitted by the brown planthopper Nilaparvata lugens Stål. In: Heong KL, Hardy B, editors. Planthoppers: new threats to the sustainability of intensive rice production systems in Asia. International Rice Research Institute, Los Baños, Philippines; 2009. p. 357-68

10. Sögawa K. The rice brown planthopper: feeding physiology and host plant interactions. Annu Rev Entomol. 1982;27:49-73.

11. Shimamoto K, Kyozuka J. Rice as a model for comparative genomics of plants. Annu Rev Plant Biol. 2002;53:399-419.

12. Zhang F, Zhu L, He G. Differential gene expression in response to brown planthopper feeding in rice. J Plant Physiol. 2004;161:53-62.

13. Wei Z, Hu W, Lin Q, Cheng X, Tong M, Zhu L, et al. Understanding rice plant resistance to the Brown Planthopper (Nilaparvata lugens): a proteomic approach. Proteomics. 2009;9:2798-808.

14. Liu C, Hao F, Hu J, Zhang W, Wan L, Zhu L, et al. Revealing different systems responses to Brown Planthopper infestation for Pest susceptible and resistant Rice plants with the combined Metabonomic and gene-expression analysis. J Proteome Res. 2010;9:6774-85.

15. Jing S, Zhao Y, Du B, Chen R, Zhu L, He G. Genomics of interaction between the brown planthopper and rice. Curr Opin Insect Sci. 2017;19:82-7.

16. Guo J, Xu C, Wu D, Zhao Y, Qiu Y, Wang X, et al. Bph6 encodes an exocystlocalized protein and confers broad resistance to planthoppers in rice. Nat Genet. 2018:50:297-306

17. Kumar K, Sarao PS, Bhatia D, Neelam K, Kaur A, Mangat GS, et al. Highresolution genetic mapping of a novel brown planthopper resistance locus, Bph34 in Oryza sativa L. X Oryza nivara (Sharma \& Shastry) derived interspecific F2 population. Theor Appl Genet. 2018;131:1163-71.

18. Qiu Y, Guo J, Jing S, Zhu L, He G. High-resolution mapping of the brown planthopper resistance gene Bph6 in rice and characterizing its resistance in the 9311 and Nipponbare near isogenic backgrounds. Theor Appl Genet. 2010;121:1601-11.

19. Boerjan B, Cardoen D, Verdonck R, Caers J, Schoofs L. Insect omics research coming of age. Can J Zool. 2012;90:440-55.

20. Oates C, Denby K, Myburg A, Slippers B, Naidoo S. Insect Gallers and their plant hosts: from omics data to systems biology. Int J Mol Sci. 2016;17:1891.

21. Liu L, Li Y, Li S, Hu N, He Y, Pong R, et al. Comparison of next-generation sequencing systems. J Biomed Biotechnol. 2012;2012:251364.

22. Rhoads A, Au KF. PacBio sequencing and its applications. Genomics Proteomics Bioinformatics. 2015;13:278-89.

23. Tilgner $H$, Grubert F, Sharon D, Snyder MP. Defining a personal, allelespecific, and single-molecule long-read transcriptome. Proc Natl Acad Sci. 2014;111:9869-74

24. Wang B, Tseng E, Regulski M, Clark TA, Hon T, Jiao Y, et al. Unveiling the complexity of the maize transcriptome by single-molecule long-read sequencing. Nat Commun. 2016;7:11708.

25. Abdel-Ghany SE, Hamilton M, Jacobi JL, Ngam P, Devitt N, Schilkey F, et al. A survey of the sorghum transcriptome using single-molecule long reads. Nat Commun. 2016;7:11706.

26. Luo Y, Ding N, Shi X, Wu Y, Wang R, Pei L, et al. Generation and comparative analysis of full-length transcriptomes in sweetpotato and its putative wild ancestor I. trifida. bioRxiv. 2017; https://doi.org/10.11 01/112425.

27. Chen S, Deng F, Jia X, Li C, Lai S. A transcriptome atlas of rabbit revealed by PacBio single-molecule long-read sequencing. Sci Rep-Uk. 2017;7:7648.

28. Ji R, Yu H, Fu Q, Chen H, Ye W, Li S, et al. Comparative transcriptome analysis of salivary glands of two populations of rice brown planthopper, Nilaparvata lugens, that differ in virulence. PLoS One. 2013;8:e79612.

29. Zhou S, Sun Z, Ma W, Chen W, Wang M. De novo analysis of the Nilaparvata lugens (Stål) antenna transcriptome and expression patterns of olfactory genes. Comp Biochem and Physiol Part D: Genomics Proteomics. 2014;9:31-9.

30. Chao Y, Yuan J, Li S, Jia S, Han L, Xu L. Analysis of Transcripts and splice isoforms in Red Clover (Trifolium pratense L.) by single-molecule long-read sequencing. BMC Plant Biol. 2018;18:300. https://doi.org/10.1186/s12870-01 8-1534-8.

31. Salmela L, Rivals E. LoRDEC: accurate and efficient long read error correction. Bioinformatics. 2014;30:3506-14.

32. Wu TD, Watanabe CK. GMAP: a genomic mapping and alignment program for mRNA and EST sequences. Bioinformatics. 2005;21:1859-75.

33. Xue J, Zhou X, Zhang C, Yu L, Fan H, Wang Z, et al. Genomes of the rice pest brown planthopper and its endosymbionts reveal complex complementary contributions for host adaptation. Genome Biol. 2014;15:521.

34. Patel AA, Steitz JA. Splicing double: insights from the second spliceosome. Nat Rev Mol Cell Biol. 2003;4:960-70, 
35. Chen M, Manley JL. Mechanisms of alternative splicing regulation: insights from molecular and genomics approaches. Nat Rev Mol Cell Biol. 2009;10:741.

36. Nilsen TW, Graveley BR. Expansion of the eukaryotic proteome by alternative splicing. Nature. 2010;463:457-63.

37. Marquez Y, Brown JWS, Simpson C, Barta A, Kalyna M. Transcriptome survey reveals increased complexity of the alternative splicing landscape in Arabidopsis. Genome Res. 2012;22:1184-95.

38. Daines B, Wang H, Wang L, Li Y, Han Y, Emmert D, et al. The Drosophila melanogaster transcriptome by paired-end RNA sequencing. Genome Res. 2011;21:315-24.

39. Ramani AK, Calarco JA, Pan Q, Mavandadi S, Wang Y, Nelson AC, et al. Genome-wide analysis of alternative splicing in Caenorhabditis elegans. Genome Res. 2011;21:342-8.

40. Wang $M$, Wang $P$, Liang F, Ye Z, Li J, Shen C, et al. A global survey of alternative splicing in allopolyploid cotton: landscape, complexity and regulation. New Phytol. 2018;217:163-78.

41. Shao W, Zhao QY, Wang XY, Xu XY, Tang Q, Li M, et al. Alternative splicing and trans-splicing events revealed by analysis of the Bombyx mori transcriptome. Rna. 2012;18:1395-407.

42. Yoon J, Abdelmohsen K, Gorospe M. Posttranscriptional gene regulation by Long noncoding RNA. J Mol Biol. 2013;425:3723-30.

43. Xiao H, Yuan Z, Guo D, Hou B, Yin C, Zhang W, et al. Genome-wide identification of long noncoding RNA genes and their potential association with fecundity and virulence in rice brown planthopper, Nilaparvata lugens. BMC Genomics. 2015;16:749.

44. Wu Y, Cheng T, Liu C, Liu D, Zhang Q, Long R, et al. Systematic identification and characterization of long non-coding RNAs in the silkworm, Bombyx mori. Plos One. 2016;11:e147147

45. Jayakodi M, Jung JW, Park D, Ahn Y, Lee S, Shin S, et al. Genome-wide characterization of long intergenic non-coding RNAs (lincRNAs) provides new insight into viral diseases in honey bees Apis cerana and Apis mellifera. BMC Genomics. 2015;16:680.

46. Young RS, Marques AC, Tibbit C, Haerty W, Bassett AR, Liu J, et al. Identification and properties of 1,119 candidate lincRNA loci in the Drosophila melanogaster genome. Genome Biol Evol. 2012;4:427-42.

47. Mercer TR, Dinger ME, Mattick JS. Long non-coding RNAs: insights into functions. Nat Rev Genet. 2009:10:155.

48. Ponting $\mathrm{CP}$, Oliver $\mathrm{PL}$, Reik W. Evolution and functions of long noncoding RNAs. Cell. 2009;136:629-41.

49. Qu Z, Adelson DL. Identification and comparative analysis of ncRNAs in human, mouse and zebrafish indicate a conserved role in regulation of genes expressed in brain. PLoS One. 2012; 7:e52275.

50. Derrien T, Johnson R, Bussotti G, Tanzer A, Djebali S, Tilgner H, et al. The GENCODE v7 catalog of human long noncoding RNAs: analysis of their gene structure, evolution, and expression. Genome Res. 2012;22:1775-89.

51. Kim D, Salzberg SL. TopHat-fusion: an algorithm for discovery of novel fusion transcripts. Genome Biol. 2011;12:R72

52. Maher CA, Kumar-Sinha C, Cao X, Kalyana-Sundaram S, Han B, Jing X, et al. Transcriptome sequencing to detect gene fusions in cancer. Nature. 2009;458:97.

53. Gao J, Fan $Y$, Wang $X$, Zhang $Y$, Pu J, Li L, et al. A conserved intronic U1 snRNP-binding sequence promotes trans-splicing in Drosophila. Genes Dev. 2015:29:760-71.

54. Nishimura A, Ashikari M, Lin S, Takashi T, Angeles ER, Yamamoto T, et al. Isolation of a Rice regeneration quantitative trait loci gene and its application to transformation systems. Proc Natl Acad Sci USA. 2005;102:11940-4.

55. Shangguan $X$, Zhang J, Liu B, Zhao $Y$, Wang H, Wang Z, et al. A mucin-like protein of Planthopper is required for feeding and induces immunity response in plants. Plant Physiol. 2018;176:552-65.

56. Roy SK, Srivastava RK, Shankar S. Inhibition of PI3K/AKT and MAPK/ERK pathways causes activation of FOXO transcription factor, leading to cell cycle arrest and apoptosis in pancreatic cancer. J Mol Signal. 2010;5:10.

57. Greer EL, Dowlatshahi D, Banko MR, Villen J, Hoang K, Blanchard D, et al. An AMPK-FOXO pathway mediates longevity induced by a novel method of dietary restriction in C. elegans. Curr Biol. 2007;17:1646-56.

58. Greer EL, Banko MR, Brunet A. AMP-activated protein kinase and FoxO transcription factors in dietary restriction-induced longevity. Ann N Y Acad Sci. 2009;1170:688-92.

59. Mátyás V, Hugo S, Felix R, Thomas R, Ernst H. The Drosophila forkhead transcription factor FOXO mediates the reduction in cell number associated with reduced insulin signaling. J Biol. 2003;2:20.
60. Kramer JM, Slade JD, Staveley BE. foxo is required for resistance to amino acid starvation in Drosophila. Genome. 2008;51:668-72.

61. Kramer JM, Davidge JT, Lockyer JM, Staveley BE. Expression of Drosophila FOXO regulates growth and can phenocopy starvation. BMC Dev Biol. 2003;3:5.

62. Zhou J, Chen X, Yan J, You K, Yuan Z, Zhou Q, et al. Brummer-dependent lipid mobilization regulates starvation resistance in Nilaparvata lugens. Arch Insect Biochem. 2018;99:e21481.

63. Huang $H$, Bao $Y$, Lao S, Huang $X, Y e ~ Y, W u$ J, et al. Rice ragged stunt virusinduced apoptosis affects virus transmission from its insect vector, the brown planthopper to the rice plant. Sci Rep-Uk. 2015;5:11413.

64. Lee JH, Jo YH, Patnaik BB, Park KB, Tindwa H, Seo GW, et al. Cloning, expression analysis, and RNA interference study of a HORMA domain containing autophagy-related gene 13 (ATG13) from the coleopteran beetle, Tenebrio molitor. Front Physiol. 2015;6:180.

65. Ghavami S, Eshragi M, Ande SR, Chazin WJ, Klonisch T, Halayko AJ, et al. S100A8/A9 induces autophagy and apoptosis via ROS-mediated cross-talk between mitochondria and lysosomes that involves BNIP3. Cell Res. 2010;20:314.

66. Deveraux QL, Reed JC. IAP family proteins-suppressors of apoptosis. Genes Dev. 1999;13:239-52

67. Duckett CS, Nava VE, Gedrich RW, Clem RJ, Van Dongen JL, Gilfillan MC, et al. A conserved family of cellular genes related to the baculovirus iap gene and encoding apoptosis inhibitors. EMBO J. 1996;15:2685-94.

68. Zhu-Salzman K, Salzman RA, Ahn J, Koiwa H. Transcriptional regulation of sorghum defense determinants against a phloem-feeding aphid. Plant Physiol. 2004;134:420-31.

69. Watson FL, Püttmann-Holgado R, Thomas F, Lamar DL, Hughes M, Kondo $M$, et al. Extensive diversity of Ig-superfamily proteins in the immune system of insects. Science. 2005;309:1874-8.

70. Legeai F, Derrien T. Identification of long non-coding RNAs in insects genomes. Curr Opin Insect Sci. 2015;7:37-44.

71. Li J, Wang J, Zeigler RS. The 3,000 rice genomes project: new opportunities and challenges for future rice research. Gigascience. 2014;3:8.

72. Denno RF, Roderick GK, Olmstead KL, Dobel HG. Density-related migration in planthoppers (Homoptera: Delphacidae): the role of habitat persistence. Am Nat. 1991;138:1513-41.

73. Arrese EL, Soulages JL. Insect fat body: energy, metabolism, and regulation. Annu Rev Entomol. 2010;55:207-25.

74. Britton JS, Edgar BA. Environmental control of the cell cycle in Drosophila: nutrition activates mitotic and endoreplicative cells by distinct mechanisms. Development. 1998;125:2149-58.

75. Subramanyam S, Smith DF, Clemens JC, Webb MA, Sardesai N, Williams CE. Functional characterization of HFR1, a high-mannose N-glycan-specific wheat lectin induced by Hessian fly larvae. Plant Physiol. 2008;147:1412-26.

76. Defagó M, Valladares G, Banchio E, Carpinella C, Palacios S. Insecticide and antifeedant activity of different plant parts of Melia azedarach on Xanthogaleruca luteola. Fitoterapia. 2006;77:500-5.

77. Baldwin IT. An ecologically motivated analysis of plant-herbivore interactions in native tobacco. Plant Physiol. 2001;127:1449-58.

78. Kliebenstein DJ, Kroymann J, Brown P, Figuth A, Pedersen D, Gershenzon J, et al. Genetic control of natural variation in Arabidopsis glucosinolate accumulation. Plant Physiol. 2001;126:811-25.

79. Mello MO, Silva-Filho MC. Plant-insect interactions: an evolutionary arms race between two distinct defense mechanisms. Braz J Plant Physiol. 2002;14:71-81.

80. Scott JG, Wen Z. Cytochromes P450 of insects: the tip of the iceberg. Pest Manag Sci. 2001;57:958-67.

81. Kim JH, Choi JS, Lee BH. PI3K/Akt and MAPK pathways evoke activation of FoxO transcription factor to undergo neuronal apoptosis in brain of the silkworm Bombyx mori (Lepidoptera: Bombycidae). Cell Mol Biol (Noisy-leGrand). 2012;Suppl 58:OL1780-85

82. Wu W, Wei W, Ablimit M, Ma Y, Fu T, Liu K, et al. Responses of two insect cell lines to starvation: autophagy prevents them from undergoing apoptosis and necrosis, respectively. J Insect Physiol. 2011;57:723-34.

83. Khoa DB, Takeda M. Expression of autophagy 8 (Atg8) and its role in the midgut and other organs of the greater wax moth, galleria mellonella, during metamorphic remodelling and under starvation. Insect Mol Biol. 2012:21:473-87.

84. Scott RC, Schuldiner O, Neufeld TP. Role and regulation of starvationinduced autophagy in the Drosophila fat body. Dev Cell. 2004;7:167-78.

85. Jing S, Liu B, Peng L, Peng $X$, Zhu L, Fu Q, et al. Development and use of EST-SSR markers for assessing genetic diversity in the brown planthopper (Nilaparvata lugens Stål). B Entomol Res. 2012;102:113-22. 
86. Wang H, Shi S, Guo Q, Nie L, Du B, Chen R, et al. High-resolution mapping of a gene conferring strong antibiosis to brown planthopper and developing resistant near-isogenic lines in 9311 background. Mol Breed. 2018;38:107.

87. Kobayashi T, Yamamoto K, Suetsugu Y, Kuwazaki S, Hattori M, Jairin J, et al. Genetic mapping of the rice resistance-breaking gene of the brown planthopper Nilaparvata lugens. Proc R Soc B Biol Sci. 2014;281:20140726.

88. Takagi H, Abe A, Yoshida K, Kosugi S, Natsume S, Mitsuoka C, et al. QTL-seq: rapid mapping of quantitative trait loci in rice by whole genome resequencing of DNA from two bulked populations. Plant J. 2013;74:174-83.

89. Hill JT, Demarest BL, Bisgrove BW, Gorsi B, Su YC, Yost HJ. MMAPPR: mutation mapping analysis pipeline for pooled RNA-seq. Genome Res. 2013; 23:687-97.

90. Liu H, Liu Q, Ge Y, Zhao Q, Zheng X, Zhao Y. hTERT promotes cell adhesion and migration independent of telomerase activity. Sci Rep-Uk. 2016;6:22886.

91. Shimizu K, Adachi J, Muraoka Y. ANGLE: a sequencing errors resistant program for predicting protein coding regions in unfinished cDNA. J Bioinforma Comput Biol. 2006;4:649-64.

92. Robinson MD, McCarthy DJ, Smyth GK. edgeR: a Bioconductor package for differential expression analysis of digital gene expression data. Bioinformatics. 2010;26:139-40.

93. Pertea M, Pertea GM, Antonescu CM, Chang T, Mendell JT, Salzberg SL. StringTie enables improved reconstruction of a transcriptome from RNA-seq reads. Nat Biotechnol. 2015;33:290-5.

\section{Publisher's Note}

Springer Nature remains neutral with regard to jurisdictional claims in published maps and institutional affiliations.

Ready to submit your research? Choose BMC and benefit from:

- fast, convenient online submission

- thorough peer review by experienced researchers in your field

- rapid publication on acceptance

- support for research data, including large and complex data types

- gold Open Access which fosters wider collaboration and increased citations

- maximum visibility for your research: over $100 \mathrm{M}$ website views per year

At $\mathrm{BMC}$, research is always in progress.

Learn more biomedcentral.com/submissions 